\title{
Stochastic Ion Acceleration by the Ion-cyclotron Instability in a Growing Magnetic Field
}

\author{
Francisco Ley ${ }_{1}^{1}$, Mario Riquelme ${ }^{2}$ (i) Lorenzo Sironi $^{3}$, Daniel Verscharen $^{4,5}$ (1) , and Astor Sandoval ${ }^{6}$ \\ ${ }^{1}$ Department of Astronomy, University of Wisconsin-Madison, USA; fley@astro.wisc.edu \\ ${ }^{2}$ Departamento de Física, Facultad de Ciencias Físicas y Matemáticas, Universidad de Chile, Chile; mario.riquelme@dfi.uchile.cl \\ ${ }^{3}$ Department of Astronomy, Columbia University, New York, NY 10027, USA; 1sironi@astro.columbia.edu \\ ${ }^{4}$ Mullard Space Science Laboratory, University College London, Dorking, UK; d.verscharen@ucl.ac.uk \\ ${ }^{5}$ Space Science Center, University of New Hampshire, Durham, NH 03824, USA \\ ${ }^{6}$ Instituto de Astrofísica, P. Universidad Católica de Chile, Chile; asandoval@astro.puc.cl \\ Received 2019 March 26; revised 2019 May 25; accepted 2019 May 28; published 2019 July 30
}

\begin{abstract}
Using 1D and 2D particle-in-cell simulations of a plasma with a growing magnetic field $\boldsymbol{B}$, we show that ions can be stochastically accelerated by the ion-cyclotron (IC) instability. As $\boldsymbol{B}$ grows, an ion pressure anisotropy $p_{\perp, i}>p_{\|, i}$ arises due to the adiabatic invariance of the ion magnetic moment $\left(p_{\|, i}\right.$ and $p_{\perp, i}$ are the ion pressures parallel and perpendicular to $\boldsymbol{B})$. When initially $\beta_{i}=0.5\left(\beta_{i} \equiv 8 \pi p_{i} /|\boldsymbol{B}|^{2}\right.$, where $p_{i}$ is the ion isotropic pressure), the pressure anisotropy is limited mainly by inelastic pitch-angle scattering provided by the IC instability, which in turn produces a nonthermal tail in the ion energy spectrum. After $\boldsymbol{B}$ is amplified by a factor of $\sim 2.7$, this tail can be approximated as a power law of index $\sim 3.4$ plus two nonthermal bumps and accounts for $2 \%-3 \%$ of the ions and $\sim 18 \%$ of their kinetic energy. On the contrary, when initially $\beta_{i}=2$, the ion scattering is dominated by the mirror instability, and the acceleration is suppressed. This implies that efficient ion acceleration requires that initially, $\beta_{i} \lesssim 1$. Although we focus on cases where $\boldsymbol{B}$ is amplified by plasma shear, we check that the acceleration occurs similarly if $\boldsymbol{B}$ grows due to plasma compression. Our results are valid in a subrelativistic regime where the ion thermal energy is $\sim 10 \%$ of the ion rest-mass energy. This acceleration process can thus be relevant in the inner region of low-luminosity accretion flows around black holes.
\end{abstract}

Key words: accretion, accretion disks - instabilities - plasmas

\section{Introduction}

Stochastic (or second-order Fermi) acceleration by plasma turbulence is considered a viable mechanism for producing nonthermal particles in several astrophysical environments. This process can, in principle, be driven by MHD plasma waves (e.g., Chandran 2003; Cho \& Lazarian 2006; Lynn et al. 2014) and kinetic plasma modes (e.g., Dermer et al. 1996; Petrosian \& Liu 2004). In this work, we use particle-in-cell (PIC) plasma simulations to show that ions can be stochastically accelerated by ion-cyclotron (IC) waves driven unstable in the presence of an ion pressure anisotropy with $p_{\perp, i}>p_{\|, i}$ (where $p_{\perp, i}$ and $p_{\|, i}$ are the ion pressures perpendicular and parallel to the local magnetic field $\boldsymbol{B}$, respectively).

The condition $p_{\perp, i} \neq p_{\|, i}$ is naturally expected in turbulent, weakly collisional plasmas. In these environments, Coulomb collisions are not able to break the adiabatic invariance of the magnetic moment $\mu_{i}$ of ions, which is defined as $\mu_{i} \equiv v_{\perp, i}^{2} / B$, where $v_{\perp, i}$ is the ion velocity perpendicular to $\boldsymbol{B}$ and $B=|\boldsymbol{B}|$. Thus, if $B$ grows (decreases), the conservation of $\mu_{i}$ will naturally produce a pressure anisotropy with $p_{\perp, i}>p_{\|, i}$ $\left(p_{\perp, i}<p_{\|, i}\right)$. Examples of weakly collisional astrophysical plasmas where the condition $p_{\perp, i} \neq p_{\|, i}$ is possible are lowluminosity accretion disks around compact objects (e.g., Sharma et al. 2006), the intracluster medium (ICM; Schekochihin et al. 2005; Lyutikov 2007), and the heliosphere (e.g., Bale et al. 2009; Maruca et al. 2011; Verscharen et al. 2019).

In these systems, the growth of ion pressure anisotropy is expected to be regulated by kinetic instabilities, which break the adiabatic invariance of $\mu_{i}$ via pitch-angle scattering of the ions. In the $p_{\perp, i}>p_{\|, i}$ regime, there are two relevant instabilities: the mirror and the IC instabilities. The mirror instability consists of nonpropagating, compressional modes, with their dominant modes having wavevectors $\boldsymbol{k}$ oblique to the direction of $\boldsymbol{B}$ (Hasegawa 1969; Southwood \& Kivelson 1993). The IC instability, on the other hand, consists of propagating electromagnetic modes, with their dominant waves having $\boldsymbol{k} \| \boldsymbol{B}$ (Anderson et al. 1991; Gary 1992). Whether the ion pitch-angle scattering is dominated by the IC or mirror instability essentially depends on how fast the instabilities grow for a given plasma regime. In this work, we show that, in a regime dominated by the IC instability, significant nonthermal ion acceleration can occur due to scattering by the IC waves.

Our study will use PIC plasma simulations to study a homogeneous plasma in which ion and electron pressure anisotropies are self-consistently produced by the continuous growth of a background magnetic field $B$. In our simulations, the magnetic field will grow on timescales significantly longer than the initial, exponential growth regime of the mirror and IC instabilities. This will allow us to capture the long-term, saturated state of the instabilities, which should be the dominant regime in astrophysical systems where $B$ experiences significant amplifications. In most of our simulations, the magnetic field will be amplified through imposing a slow shear motion in the plasma, which will increase $B$ due to magnetic flux conservation. However, we will also use simulations of compressing plasmas to show that our main results are fairly independent of the specific mechanism that drives the growth of $B$. In this study, we focus on conditions applicable to the inner regions of low-luminosity accretion flows around black holes. This will be done by assuming in all of our runs a hot plasma with initially equal ion and electron temperatures $\left(T_{i}=T_{e}\right.$ ) and with $k_{B} T_{i} / m_{i} c^{2}=0.05$ (where $k_{B}$ is the Boltzmann constant, $m_{i}$ is the mass of the ions, and $c$ is the speed of light). 


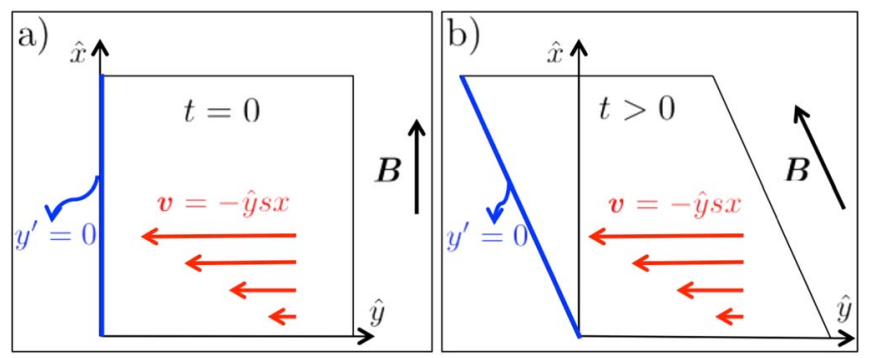

Figure 1. Panels (a) and (b) show a sketch of the simulation domain in our 2D shearing simulations at $t=0$ and $t>0$, respectively. The 2D domain follows the shearing flow of the plasma (red arrows), acquiring a parallelogram shape. Magnetic flux conservation changes the magnitude and orientation of the background magnetic field $\boldsymbol{B}$, which is always parallel to the nonhorizontal sides of the parallelogram. The blue lines show the domain of our 1D runs.

Our paper is organized as follows. In Section 2 we present our simulation method and setup. In Section 3 we use 2D simulations to show that ions can be accelerated by the IC instability under the condition that initially, $\beta_{i} \lesssim 1$ $\left(\beta_{i} \equiv 8 \pi p_{i} /|\boldsymbol{B}|^{2}\right.$, where $p_{i}$ is the ion isotropic pressure). In Section 4 we use 1D simulations to clarify the role of the IC and mirror modes in the acceleration, as well as to show that our results are independent of the rate at which $B$ is amplified and the numerical ion-to-electron mass ratio, $m_{i} / m_{e}$. In Section 5 we describe the acceleration in further detail, connecting the growth of IC modes of different wavenumber $k$ with the acceleration of ions of different energy. In Section 6 we show that our results are fairly independent of whether $B$ is amplified via plasma shear or compression. In Section 7 we summarize our results and present our conclusions. Additionally, in Appendix A we provide details on the implementation of our 1D simulations, and in Appendix B we use linear theory to analyze the applicability of our simulation results to realistic astrophysical environments.

\section{Simulation Setup}

We use the PIC code TRISTAN-MP (Buneman 1993; Spitkovsky 2005) to simulate both a shearing and a compressing plasma made of ions and electrons. In the shearing case, the plasma is initially in the presence of a homogeneous initial magnetic field that points along the $x$-axis, $\boldsymbol{B}=B_{0} \hat{x}$. This field is amplified by imposing a shear plasma velocity $\boldsymbol{v}=-s x \hat{y}$ (represented by red arrows in Figure 1(a)), where $x$ is the distance along $\hat{x}$ and $s$ is the shear rate. This way, the background magnetic field $\boldsymbol{B}$ in the simulation permanently increases and changes direction due to magnetic flux conservation, with its $y$-component evolving as $d B_{y} / d t=-s B_{0}$, while $d B_{x} / d t=$ $d B_{z} / d t=0$ (Figure 1(b) shows how $\boldsymbol{B}$ changes orientation for $t>0)$. Due to $\mu_{j}$ conservation, this magnetic growth drives $p_{\perp, j}>p_{\|, j}$ during the whole simulation, allowing the triggering of kinetic instabilities that limit the pressure anisotropies.

Our 2D shearing runs use initially square simulation domains (as depicted in Figure 1(a)) that follow the mean shear motion of the plasma. Therefore, the 2D domain acquires a parallelogram shape for $t>0$ (as shown in Figure 1(b)). The positions of the plasma particles are therefore given in terms of the so-called "shearing coordinates," which are described both in Appendix A of this paper and in the Appendix of Riquelme et al. (2012). In our 1D shearing runs, on the other hand, the simulation domain corresponds to the blue lines shown in Figures 1(a) and (b), which also move with the shearing flow.
Table 1

Parameters of the Simulations

\begin{tabular}{lccccc}
\hline \hline Runs & $\begin{array}{c}\omega_{c, i}^{\text {init }} / s \\
\left(\text { or } \omega_{c, i}^{\text {init }} / q\right)\end{array}$ & $m_{i} / m_{e}$ & $\beta_{i}^{\text {init }}$ & $N_{\mathrm{ppc}}$ & $L / R_{L, i}^{\text {init }}[\mathrm{D}]$ \\
\hline S2m2b0.5 & 800 & 2 & 0.5 & 160 & $60[2]$ \\
S2m2b2 & 800 & 2 & 2 & 160 & $60[2]$ \\
S2m10b0.5 & 800 & 10 & 0.5 & 160 & $60[2]$ \\
S2m10b2 & 800 & 10 & 2 & 160 & $60[2]$ \\
S1m2b0.5 & 800 & 2 & 0.5 & 640 & $150[1]$ \\
S1m2b2 & 800 & 2 & 2 & 640 & $150[1]$ \\
S1m8b0.5 & 800 & 8 & 0.5 & 640 & $150[1]$ \\
S1m32b0.5 & 800 & 32 & 0.5 & 640 & $150[1]$ \\
S1m128b0.5 & 800 & 128 & 0.5 & 1280 & $150[1]$ \\
S1m8b0.5b & 400 & 8 & 0.5 & 640 & $150[1]$ \\
S1m8b0.5c & 1600 & 8 & 0.5 & 640 & $150[1]$ \\
S1m8b0.5d & 3200 & 8 & 0.5 & 3600 & $150[1]$ \\
C1m8b0.5a & 1600 & 8 & 0.5 & 3600 & $190[1]$ \\
C1m8b0.5b & 3200 & 8 & 0.5 & 3600 & $190[1]$ \\
C1m16b0.5a & 1600 & 16 & 0.5 & 3600 & $190[1]$ \\
C1m16b0.5b & 3200 & 16 & 0.5 & 3600 & $190[1]$ \\
\hline
\end{tabular}

Note. Simulation parameters: the initial cyclotron frequency of the ions $\omega_{c, i}^{\text {init }}$ (in units of $s$ in the shearing runs and $q$ in the compressing runs), $m_{i} / m_{e}, \beta_{i}^{\text {init }}$, the number of particles per cell $N_{\mathrm{ppc}}$ (considering ions and electrons), and the initial box size in units of the initial ion Larmor radius $L / R_{L, i}^{\text {init }}$ (with the number of dimensions $\mathrm{D}$ in brackets). In $2 \mathrm{D}, L$ corresponds to the height and width of the box. In all runs, initially, $k_{B} T_{i} / m_{i} c^{2}=0.05, T_{e}=T_{i}$, the electron skin depth $c / \omega_{p, e} / \Delta_{x}=15$ (where $\Delta_{x}$ is the grid point separation), and the speed of light $c=0.225 \Delta_{x} / \Delta_{t}$ (shearing runs) and $0.15 \Delta_{x} / \Delta_{t}$ (compressing runs), where $\Delta_{t}$ is the simulation time step.

Since the symmetry axis of this 1D domain is permanently parallel to $\boldsymbol{B}$, our 1D approach allows us to capture waves that propagate parallel to $\boldsymbol{B}$. The self-consistent implementation of the $1 \mathrm{D}$ runs requires a small change in the definition of our shear coordinates, which is explained in detail in Appendix A. In Section 4.1 we show that our 1D runs give essentially the same results as our 2D runs as long as the dominant instabilities produce modes parallel to $\boldsymbol{B}$, such as the IC instability.

In our compressing plasma runs, on the other hand, the simulation box is compressed along the two directions perpendicular to the background field $\boldsymbol{B}$, producing the permanent growth of both $\boldsymbol{B}$ and $p_{\perp, j} / p_{\|, j}$. For this, we use the same setup as in Sironi \& Narayan (2015). In this case, $\boldsymbol{B}$ evolves as $\boldsymbol{B}=\hat{x} B_{0}(1+q t)^{2}$, where the constant $q$ provides the timescale for the plasma compression.

Our plasma parameters are the initial temperature of ions and electrons $\left(T_{i}\right.$ and $\left.T_{e}\right)$, the initial ratio between ion pressure and magnetic pressure $\left(\beta_{i}^{\text {init }}\right)$, the ion-to-electron mass ratio $m_{i} / m_{e}$, and the ion "magnetization," which is defined as the ratio between the initial cyclotron frequency of the ions $\left(\omega_{c, i}^{\text {init }}\right)$ and $s$ (for shearing plasma runs) or $q$ (for compressing plasma runs). The initial cyclotron frequency of the ions is defined as $\omega_{c, i}^{\text {init }}=e B_{0} / m_{i} c$, with $e$ and $B_{0}$ being the magnitude of the electron and ion electric charges and the initial magnetic field.

As mentioned above, all of our shearing and compressing simulations initially have $T_{i}=T_{e}$ and $k_{B} T_{i} / m_{i} c^{2}=0.05$. Also, these runs use $m_{i} / m_{e}$ and ion magnetizations much smaller than expected in real astrophysical settings. ${ }^{7}$ Because of this, the dependence of the ion acceleration on these

\footnotetext{
7 For instance, at $\sim 10$ Schwarzschild radii from the supermassive black hole Sgr A*, one expects $\omega_{c, i} / s \sim 10^{8}$ (e.g., Ponti et al. 2017), where we have approximated $s$ as the Keplerian angular frequency at that radius.
} 


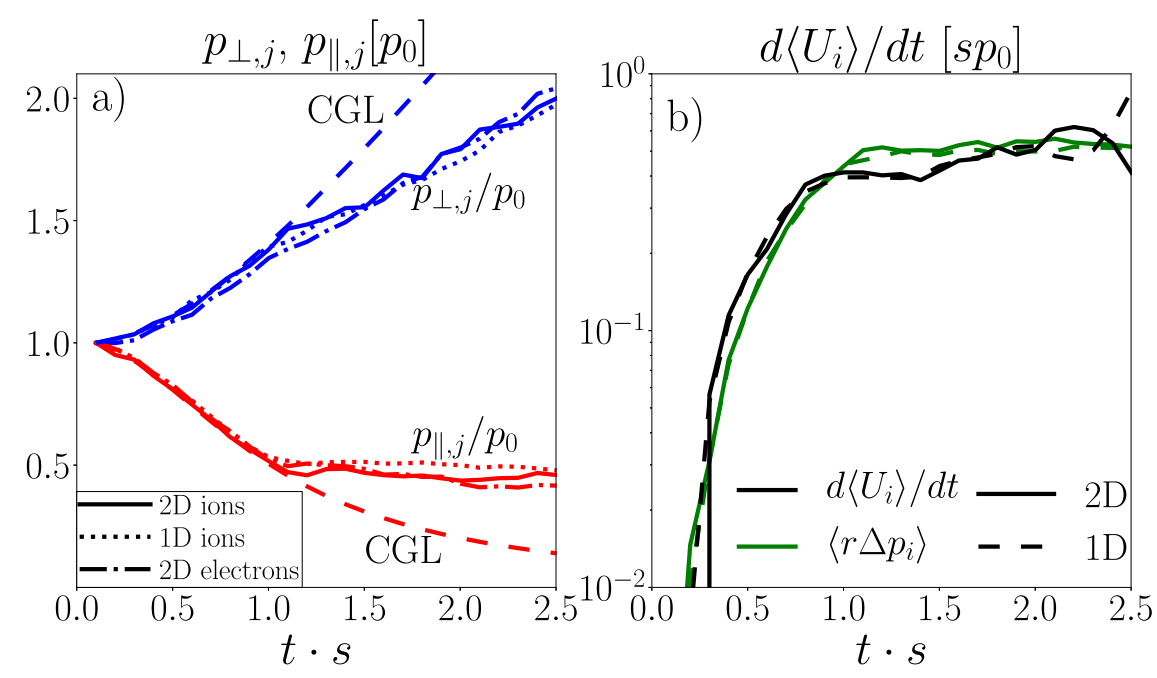

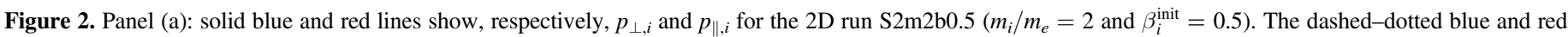

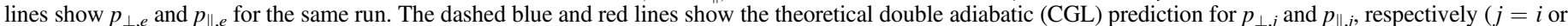

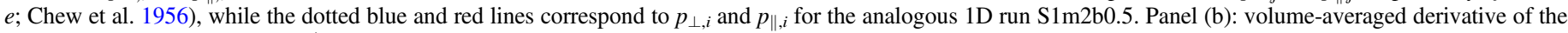

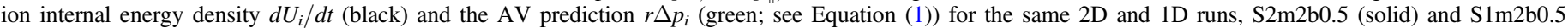
(dashed). Both $d U_{i} / d t$ and $r \Delta p_{i}$ evolve very similarly in $1 \mathrm{D}$ and $2 \mathrm{D}$, and in both cases, the ion energy gain reproduces the AV prediction fairly well.

parameters will be carefully assessed, with our main conclusion being that neither $m_{i} / m_{e}$ nor $\omega_{c, i}^{\text {init }} / s$ (or $\omega_{c, i}^{\text {init }} / q$ ) play a significant role.

The numerical parameters in our runs are the number of macroparticles per cell $\left(N_{\mathrm{ppc}}\right)$, the electron skin depth in terms of grid point spacing $\left(c / \omega_{p, e} / \Delta_{x}\right.$, where $\omega_{p, e}^{2}=4 \pi n_{e} e^{2} / m_{e}$ is the square of the electron plasma frequency and $n_{e}$ is the electron number density), and the box size in terms of the initial ion Larmor radius $\left(L / R_{L, i}^{\text {init. }} ; \quad R_{L, i}^{\text {init }}=v_{\mathrm{th}, i} / \omega_{c, i}^{\text {init }}, \quad\right.$ where $\left.v_{\mathrm{th}, i}^{2}=k_{B} T_{i} / m_{i}\right)$. Table 1 shows a summary of our key simulations. We ran a series of simulations ensuring that the numerical parameters do not affect our results. The runs used just for numerical convergence are not in Table 1.

\section{Ion Acceleration by the IC Instability}

We use 2D shearing plasma simulations to show that ions can be stochastically accelerated by the IC modes. First, we show the example of runs dominated by the IC instability (with $\left.\beta_{i}^{\text {init }}=0.5\right)$, demonstrating that in this case, a prominent nonthermal tail appears. Then, using simulations with $\beta_{i}^{\text {init }}=2$, we show that for $\beta_{i}^{\text {init }} \gtrsim 1$, the mirror instability dominates, with a corresponding suppression of the accelerating effect of the IC modes. We use 2D simulations with $m_{i} / m_{e}=2$ and 10 to show that, as far as the ion physics is concerned, our results are fairly independent of the value of $m_{i} / m_{e}$.

\subsection{IC-versus Mirror-dominated Regimes}

With solid blue and red lines, Figure 2(a) shows the respective evolutions of $p_{\perp, i}$ and $p_{\|, i}$ for run $\mathrm{S} 2 \mathrm{~m} 2 \mathrm{~b} 0.5$, which uses $\beta_{i}^{\text {init }}=0.5, m_{i} / m_{e}=2$, and $\omega_{c, i}^{\text {init }} / s=800$. We see that until $t \cdot s \approx 1$, the evolutions of both $p_{\perp, i}$ and $p_{\|, i}$ are in agreement with the "double adiabatic" prediction (dashed lines; Chew et al. 1956), which is due to the conservation of $\mu_{i}$ and the second adiabatic invariant. At $t \cdot s \gtrsim 1$, the adiabatic evolution of $p_{\perp, i}$ and $p_{\|, i}$ is broken by the appearance of ion pressure anisotropy instabilities, which produce rapid pitchangle scattering of the ions. The electron pressures $p_{\perp, e}$ and $p_{\|, e}$ evolve similarly to the ion case, as shown by the dashed-dotted blue and red lines in Figure 2(a). The break in the adiabatic evolution of the electrons is caused by the pitch-angle scattering provided by the whistler instability, as we will show in Section 5.2.

Figure 3 shows that the ion pitch-angle scattering in run $\mathrm{S} 2 \mathrm{~m} 2 \mathrm{~b} 0.5$ is dominated by the IC instability. Indeed, Figures 3(a)-(c) show a snapshot at $t \cdot s=2$ of the three components of $\delta \boldsymbol{B}$ (where $\delta \boldsymbol{B} \equiv \boldsymbol{B}-\langle\boldsymbol{B}\rangle$ and \langle\rangle denotes an average over the entire box volume). We see that $\delta B_{x}$ and $\delta B_{z}$ are the largest components of $\delta \boldsymbol{B}$. And, considering that the black arrows represent the direction of $\langle\boldsymbol{B}\rangle$, we see that $\delta \boldsymbol{B}_{x}$ and $\delta B_{z}$ (and, therefore, $\delta \boldsymbol{B}$ ) are dominated by nearly parallel modes, which are consistent with the presence of transverse, circularly polarized IC modes. The whistler modes are also nearly parallel and should appear in $\delta B_{x}$ and $\delta B_{z}$ as well. However, their amplitude is expected to be less significant than that of the IC modes, due to the smaller mass of the electrons relative to the ions. ${ }^{8}$ Here $\delta B_{y}$ shows a mixture of the nearly parallel modes, plus oblique (nearly perpendicular) modes, which are consistent with the presence of mirror modes. Indeed, these modes mainly contribute to the $\delta \boldsymbol{B}$ components parallel to the plane of the simulation (they show no $\delta B_{z}$ component), which is in line with the expectation that $\delta \boldsymbol{B}$ of the mirror modes is nearly perpendicular to $\boldsymbol{k} \times \boldsymbol{B}$ (Pokhotelov et al. 2004).

The presence of the nearly parallel IC modes can also be seen from the fluctuations in the electric field, $\delta \boldsymbol{E}$, shown in Figures 3(d)-(f). ${ }^{9}$ This electric field is expected, since the IC modes have finite phase velocities, $v_{\phi}$, which are related to $\delta \boldsymbol{E}$

\footnotetext{
8 This is because, for the resonant interaction between ions (electrons) and IC modes (whistler modes), the effective ion (electron) scattering rate is expected to be $\nu_{\text {eff } i} \sim \omega_{c, i} \delta B^{2} / B^{2}$ ( $\nu_{\text {eff }, e} \sim \omega_{c, e} \delta B^{2} / B^{2}$; see, e.g., Riquelme et al. 2016). Thus, given that in this setup, one expects $\nu_{\text {eff, } i} \sim \nu_{\text {eff, } e} \sim s$ (see discussion in Section 4.3), the contribution of the whistler modes to $\delta B_{x}^{2}$ and $\delta B_{z}^{2}$ in run $\mathrm{S} 2 \mathrm{~m} 2 \mathrm{~b} 0.5$ should be $\sim$ two times smaller than that of the IC modes.

9 Since our simulations are performed in the "shearing coordinate" frame (Riquelme et al. 2012), there is no electric field associated with the large-scale shearing motion of the plasma. Therefore, $\delta \boldsymbol{E}$ corresponds to the entire electric field present in the simulation.
} 

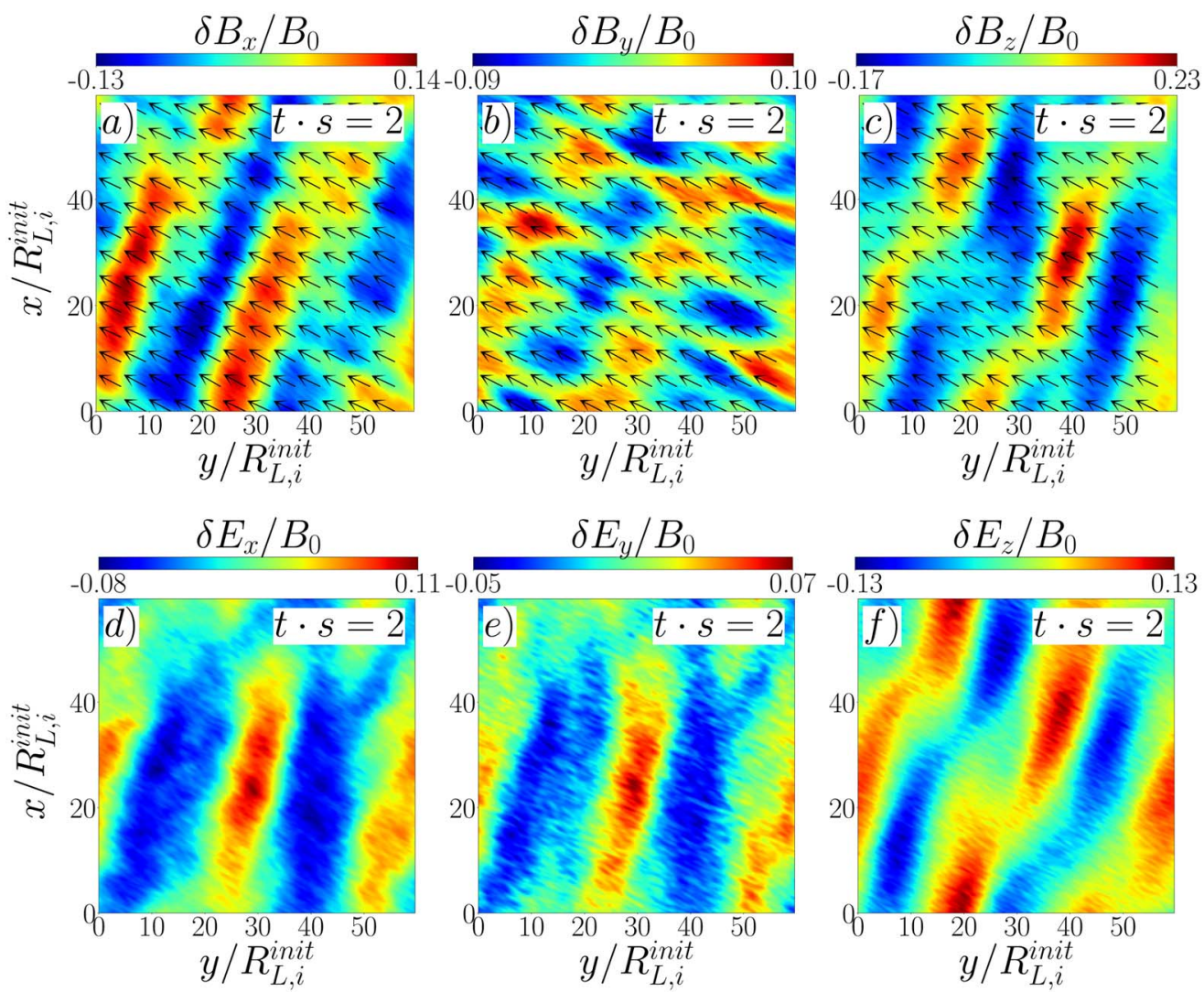

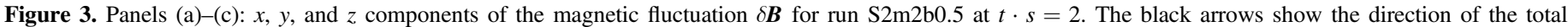
magnetic field $\boldsymbol{B}$. Panels (d)-(f): same components but for the electric field fluctuation $\delta \boldsymbol{E}$.

by $|\delta \boldsymbol{E}|=|\delta \boldsymbol{B}| v_{\phi} / c$ (this is a consequence of Faraday's law applied to the transverse IC modes). The mirror modes, on the other hand, are "purely growing" (see, e.g., Southwood \& Kivelson 1993), which means that their phase velocity vanishes. This implies that, as we see in Figure 3, no electric field associated with the subdominant mirror modes should be present.

The dominance of the IC modes in run $\mathrm{S} 2 \mathrm{~m} 2 \mathrm{~b} 0.5$ can also be inferred from the Fourier transforms (FTs) of the three components of $\delta \boldsymbol{B}\left(\mathrm{FT}\left(\delta B_{x}\right), \mathrm{FT}\left(\delta B_{y}\right)\right.$, and $\left.\mathrm{FT}\left(\delta B_{z}\right)\right)$, shown in Figure 4. Figures $4(\mathrm{a})$ and (c) show that essentially all of the power in $\mathrm{FT}\left(\delta B_{x}\right)$ and $\mathrm{FT}\left(\delta \boldsymbol{B}_{z}\right)$ is well aligned with the direction of the mean magnetic field $\langle\boldsymbol{B}\rangle$ (shown by the solid black line), which is consistent with $\delta B_{x}$ and $\delta B_{z}$ being dominated by the IC modes, with a smaller contribution from the whistler waves. ${ }^{10}$ On the other hand, Figure 4(b) shows that the contribution of the oblique (nearly perpendicular) mirror modes to $\mathrm{FT}\left(\delta B_{y}\right)$ is comparable to that of the IC modes. However, since $\delta B_{x}$ and $\delta B_{z}$ are the largest components of $\delta \boldsymbol{B}$ (as seen in Figure 3), our Fourier analysis confirms that the IC instability contributes the most to $\delta \boldsymbol{B}$.

This scenario, however, changes in the $\beta_{i}^{\text {init }} \gtrsim 1$ regime, in which the mirror instability becomes dominant. The transition from IC-dominated to mirror-dominated regimes can be seen by directly comparing simulations that only differ in their $\beta_{i}^{\text {init }}$. Figure 5(a) shows (solid lines) the magnetic energy of $\delta \boldsymbol{B}$ along

\footnotetext{
${ }^{10}$ Because of the small-scale separation between ions and electrons in run S2m2b0.5, Figures 4(a) and (c) do not allow for distinguishing the contributions from the IC and whistler modes. This distinction, however, can be achieved by performing FTs of the fields in time and space, which is done in Section 5.2 using 1D simulations.
}

different axes as a function of time for run $\mathrm{S} 2 \mathrm{~m} 2 \mathrm{~b} 0.5$ $\left(m_{i} / m_{e}=2\right.$ and $\left.\beta_{i}^{\text {init }}=0.5\right)$. This energy is expressed in terms of the $\delta \boldsymbol{B}$ components parallel to $\langle\boldsymbol{B}\rangle\left(\delta \boldsymbol{B}_{\|}\right.$; blue), perpendicular to $\langle\boldsymbol{B}\rangle$ but parallel to the plane of the simulation $\left(\delta B_{x y, \perp}\right.$; red), and perpendicular to both $\langle\boldsymbol{B}\rangle$ and the plane of the simulation $\left(\delta B_{z}\right.$; green). During most of the simulation, the energy of the magnetic fluctuations is indeed contained mainly in $\delta B_{x y, \perp}$ and $\delta B_{z}$, implying that the IC modes have the largest amplitude during most of the simulation time. By the end of the run $(t \cdot s=2.5)$, however, $\delta B_{\|}^{2}$ becomes comparable to $\delta B_{x y, \perp}^{2}$ and $\delta B_{z}^{2}$, implying that in the long-term, the mirror fluctuations can still reach amplitudes comparable to the IC modes. We have thus decided to concentrate on the regime where the IC instability clearly dominates the pitch-angle scattering of the ions by running the simulations until $t \cdot s=2.5$ (thus with a maximum $B$ amplification factor of $\sim 2.7$ ).

Figure 5(b), on the other hand, shows the evolution of the same magnetic energy components for run $\mathrm{S} 2 \mathrm{~m} 2 \mathrm{~b} 2$ $\left(m_{i} / m_{e}=2\right.$ and $\left.\beta_{i}^{\text {init }}=2\right)$. In this run, the ions are under the same conditions as in run $\mathrm{S} 2 \mathrm{~m} 2 \mathrm{~b} 0.5$ but with a smaller initial background magnetic field, so that $\beta_{i}^{\text {init }}=2$. We see that in this case, $\delta B_{z}$ and $\delta B_{x y, \perp}$ are subdominant in the saturated stage of the instabilities, and the energy in the magnetic fluctuations is dominated by $\delta B_{\|}{ }^{2}$. This result indicates that the oblique mirror modes are more prominent than the IC modes in this case, with the transition from IC-dominated to mirror-dominated regimes happening at $\beta_{i}^{\text {init }} \sim 1$.

The dominance of the mirror modes in run $\mathrm{S} 2 \mathrm{~m} 2 \mathrm{~b} 2$ can also be seen from the FT of the three $\delta \boldsymbol{B}$ components at $t \cdot s=2$, which we show in Figure 6 . In this case, $\operatorname{FT}\left(\delta B_{x}\right)$ and $\operatorname{FT}\left(\delta B_{y}\right)$ 

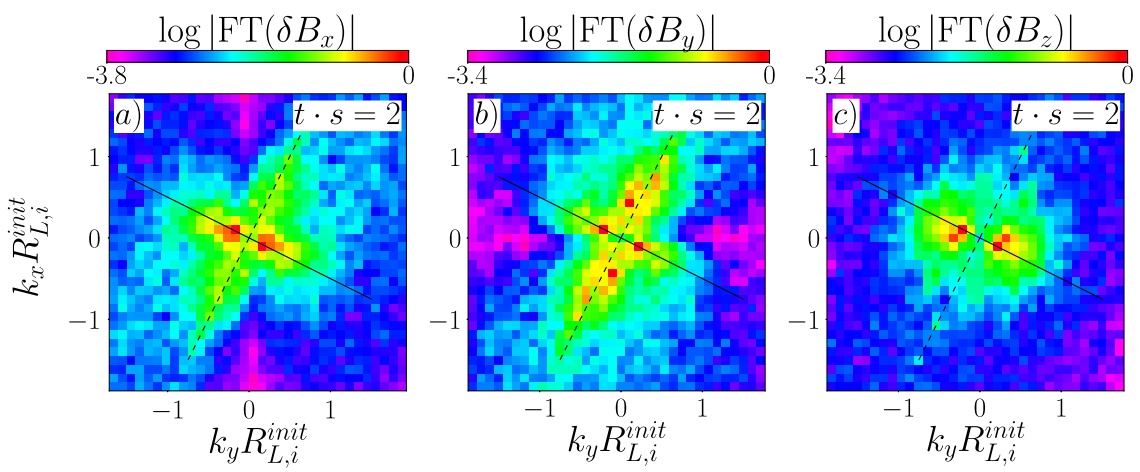

Figure 4. Magnitude of the FTs of $\delta B_{x}, \delta B_{y}$, and $\delta B_{z}$ for run $\mathrm{S} 2 \mathrm{~m} 2 \mathrm{~b} 0.5$ at $t \cdot s=2$. The three FTs are normalized by their maximum values. The solid and dotted black lines indicate the directions parallel and perpendicular to the background magnetic field.
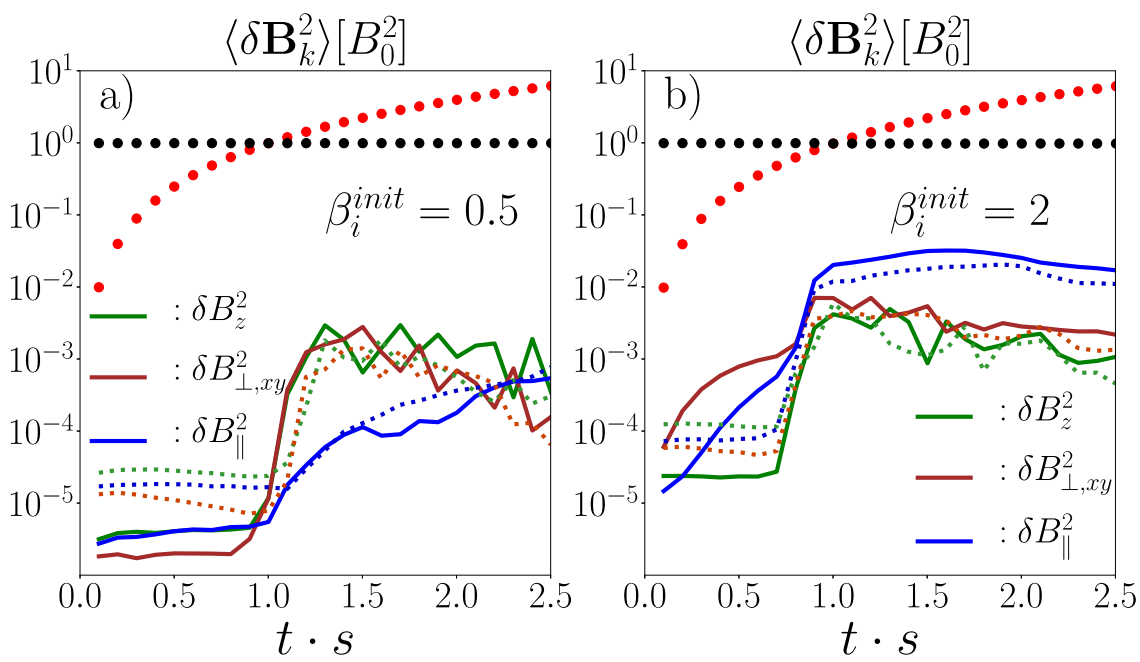

Figure 5. The solid lines show the volume-averaged magnetic energy of $\delta \boldsymbol{B}$ along different axes and as a function of time for runs $\mathrm{S} 2 \mathrm{~m} 2 \mathrm{~b} 0.5\left(m_{i} / m_{e}=2\right.$ and $\beta_{i}^{\text {init }}=0.5$; panel (a)) and $\mathrm{S} 2 \mathrm{~m} 2 \mathrm{~b} 2\left(m_{i} / m_{e}=2\right.$ and $\beta_{i}^{\text {init }}=2$; panel (b)). Here $\delta B_{\|}$(blue) is the component parallel to $\langle\boldsymbol{B}\rangle$, and $\delta \boldsymbol{B}_{x y, \perp}$ (red) and $\delta \boldsymbol{B}_{z}$ (green) are the components perpendicular to $\langle\boldsymbol{B}\rangle$ but, respectively, parallel and perpendicular to the simulation plane. The thick dotted black and red lines represent the volumeaveraged values of $\left\langle B_{x}\right\rangle^{2}$ and $\left\langle B_{y}\right\rangle^{2}$, respectively, which show how $\langle\boldsymbol{B}\rangle$ grows during the simulation. The thin dotted lines show the same quantities but for runs $\mathrm{S} 2 \mathrm{~m} 10 \mathrm{~b} 0.5\left(m_{i} / m_{e}=10\right.$ and $\beta_{i}^{\text {init }}=0.5$; panel (a) $)$ and S2m10b2 $\left(m_{i} / m_{e}=10\right.$ and $\beta_{i}^{\text {init }}=2$; panel (b)).
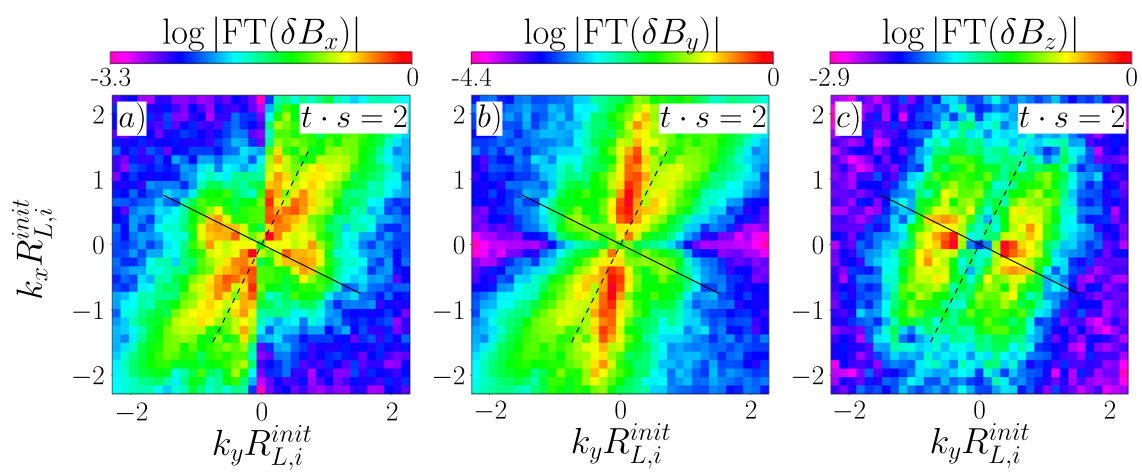

Figure 6. Same as Figure 4 but for run $\mathrm{S} 2 \mathrm{~m} 2 \mathrm{~b} 2$.

are dominated by the oblique mirror modes, ${ }^{11}$ and $\mathrm{FT}\left(\delta B_{z}\right)$ is still dominated by the nearly parallel IC modes, which is consistent with the expectation that the magnetic fluctuations caused by the mirror modes should be nearly parallel to the plane of the simulation (Pokhotelov et al. 2004). However, the

\footnotetext{
${ }^{11}$ Figure 6(b) shows a prevalence of the mirror modes quasi-parallel to the $x$ axis. This is likely due to the action of the shear, which, due to magnetic flux freezing, may favor the growth of $\delta \boldsymbol{B}$ components parallel to the shear velocity.
}

fact that $\delta B_{z}$ is the smallest component of $\delta \boldsymbol{B}$ (as seen in Figure 5(b)) implies that in run $\mathrm{S} 2 \mathrm{~m} 2 \mathrm{~b} 2$, the mirror modes contribute the most to $\delta \boldsymbol{B}$.

In order to explore the sensitivity of these results to $m_{i} / m_{e}$, in Figures 5(a) and (b), we overplot $\delta B_{\|}^{2}, \delta B_{x y, \perp}$, and $\delta B_{z}$ for simulations S2m10b0.5 and S2m10b2, which have the same ion conditions as in runs $\mathrm{S} 2 \mathrm{~m} 2 \mathrm{~b} 0.5$ and $\mathrm{S} 2 \mathrm{~m} 2 \mathrm{~b} 2$ (i.e., the same values of $\beta_{i}^{\text {init }}, k_{B} T_{i} / m_{i} c^{2}$, and $\left.\omega_{c, i}^{\text {init }} / s\right)$ but with $m_{i} / m_{e}=10$ instead of $m_{i} / m_{e}=2$. We see that for the two $\beta_{i}^{\text {init }}$, the 

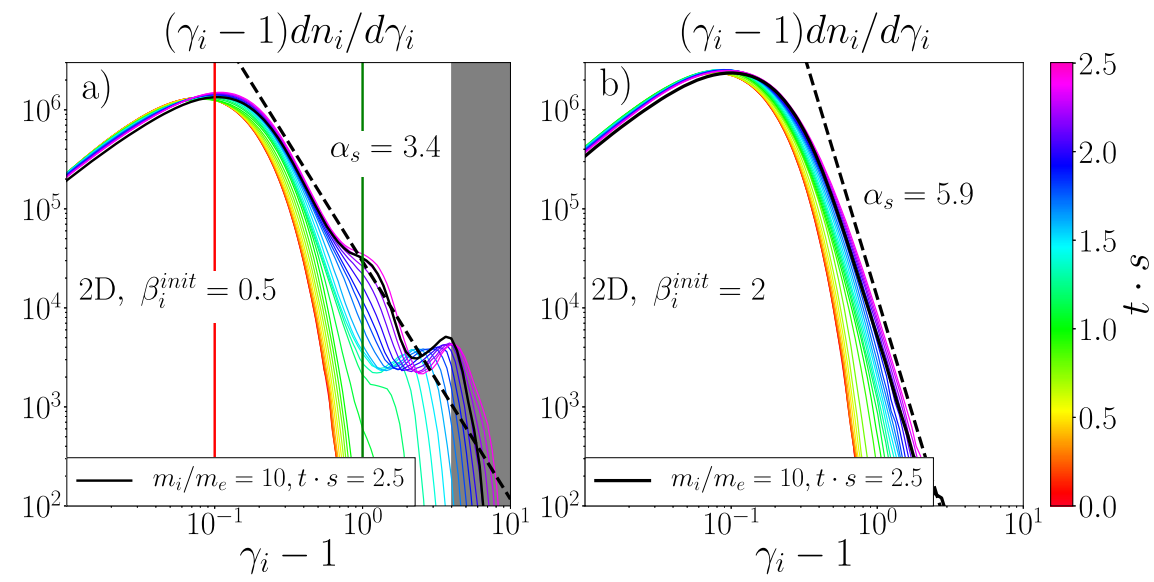

Figure 7. Evolution of the ion spectra for runs $\mathrm{S} 2 \mathrm{~m} 2 \mathrm{~b} 0.5\left(m_{i} / m_{e}=2, \beta_{i}^{\text {init }}=0.5\right.$; panel (a)) and $\mathrm{S} 2 \mathrm{~m} 2 \mathrm{~b} 2\left(m_{i} / m_{e}=2, \beta_{i}^{\text {init }}=2\right.$; panel (b)), which correspond to regimes dominated by the IC and mirror instabilities, respectively. The color bar indicates the time for each spectrum. The overplotted black lines correspond to the final spectra of runs S2m10b0.5 $\left(m_{i} / m_{e}=10, \beta_{i}^{\text {init }}=0.5\right.$; panel (a)) and S2m10b2 $\left(m_{i} / m_{e}=10, \beta_{i}^{\text {init }}=2\right.$; panel (b)), which, apart from using different mass ratios, assume the same plasma conditions as runs $\mathrm{S} 2 \mathrm{~m} 2 \mathrm{~b} 0.5$ and $\mathrm{S} 2 \mathrm{~m} 2 \mathrm{~b} 2$, respectively.

evolutions of $\delta B_{x y, \perp}, \delta B_{z}$, and $\delta B_{\|}$are fairly independent of $m_{i} / m_{e}$. Thus, the mass ratio does not appear to significantly affect the relative importance of the IC and mirror instabilities in the different $\beta_{i}^{\text {init }}$ regimes.

This result also implies that the effect of the electron pressure anisotropy on the mirror and IC instabilities is captured well by our runs. Indeed, although the electron pressure anisotropy is expected to mainly drive the growth of whistler modes (Gary \& Wang 1996), it can also have an effect in reducing (increasing) the growth rates of the IC (mirror) modes (Remya et al. 2013; Riquelme et al. 2016). The fact that in our runs, the dominance of the IC instability for $\beta_{i} \lesssim 1$ occurs similarly for $m_{i} / m_{e}=2$ and 10 implies that this effect is also fairly independent of the value of $m_{i} / m_{e}$.

Additionally, in Appendix B we use linear theory calculations to show that the condition $\beta_{i}^{\text {init }} \lesssim 1$ for the dominance of the IC instability should continue to hold even in realistic astrophysical plasma conditions, with $m_{i} / m_{e}=1836, \omega_{c, i}^{\text {init }} / s \gg 800$, and $T_{e}=T_{i}$. In the next section, we show that this IC dominance also results in a significant nonthermal ion acceleration, which is strongly suppressed when the mirror modes dominate.

\subsection{Ion Heating and Acceleration}

It is well known that in a collisionless, shearing plasma, the particles are heated by the so-called "anisotropic viscosity" (AV). Indeed, for a homogeneous plasma subject to shear, the internal energy density for species $j, U_{j}$, evolves as (Kulsrud 1983; Snyder et al. 1997)

$$
\frac{d U_{j}}{d t}=r \Delta p_{j},
$$

where $r$ is the growth rate of the magnetic field $(r \equiv(d B / d t) /$ $B)$. Equation (1) is fairly well reproduced in our simulations, as can be seen from Figure 2(b), which shows the volumeaveraged heating rate of ions $d U_{i} / d t$ (solid black) and $r \Delta p_{i}$ (solid green) for run $\mathrm{S} 2 \mathrm{~m} 2 \mathrm{~b} 0.5$.

Since $d U_{i} / d t$ is dominated by the ion pressure anisotropy, the ion heating is ultimately regulated by the pitch-angle scattering provided by either the IC or mirror instabilities. In this section, we show that when this scattering is provided mainly by the IC modes, it can also give rise to significant stochastic ion acceleration.
Figure 7(a) shows the evolution of the ion spectrum for run $\mathrm{S} 2 \mathrm{~m} 2 \mathrm{~b} 0.5\left(\beta_{i}^{\text {init }}=0.5, m_{i} / m_{e}=2\right)$, with the color bar indicating the time for each spectrum. This simulation shows the rapid growth of a nonthermal tail that starts once the IC instability grows and saturates $(t \cdot s \sim 1$, as seen in Figure 5(a)). By $t \cdot s=2.5$, the tail can be approximated by a power law $d n_{i} / d \gamma_{i} \propto\left(\gamma_{i}-1\right)^{-\alpha_{s}}$ with spectral index $\alpha_{s} \approx 3.4$ plus two bumps ( $\gamma_{i}$ is the ion Lorentz factor). The nonthermal tail at $t \cdot s=2.5$ reaches Lorentz factors $\gamma_{i} \sim 10$ and contains $\sim 2 \%-3 \%$ of the ions and $\sim 18 \%$ of their energy. ${ }^{12}$ The solid black line in Figure 7(a) represents the final $(t \cdot s=2.5)$ spectra for the analogous run $\mathrm{S} 2 \mathrm{~m} 10 \mathrm{~b} 0.5$ (with $m_{i} / m_{e}=10$ instead of 2). The small difference between the $m_{i} / m_{e}=2$ and 10 cases shows that, as long as the ion parameters $k_{B} T_{i} / m_{i} c^{2}, \beta_{i}^{\text {init }}$, and $\omega_{c, i}^{\text {init }} / s$ are the same, the ionto-electron mass ratio does not play a significant role in determining the ion acceleration efficiency. The independence of the acceleration mechanism on $m_{i} / m_{e}$, as well as on $\omega_{c, i}^{\text {init }} / s$, will be further tested using 1D simulations in Section 4.

Figure 7(b) shows the evolution of the ion spectra for the 2D run $\mathrm{S} 2 \mathrm{~m} 2 \mathrm{~b} 2\left(m_{i} / m_{e}=2\right.$ and $\left.\beta_{i}^{\text {init }}=2\right)$. In this case, the growth of the nonthermal energy tail is also present, but with a slower growth throughout the whole simulation. By $t \cdot s=2.5$, the tail can be approximated by a much less pronounced power law with spectral index $\alpha_{s} \approx 5.9$. The solid black line represents the final spectrum for the analogous run $\mathrm{S} 2 \mathrm{~m} 10 \mathrm{~b} 2$, with $m_{i} / m_{e}=10$. The small difference between the $m_{i} / m_{e}=2$ and 10 cases suggests that, as in the IC-dominated case, the ion-to-electron mass ratio is fairly unimportant in determining the ion acceleration efficiency.

These results strongly suggest that the presence of IC modes is key for the acceleration of ions. In Section 4 we show that this is indeed the case, making use of 1D simulations in which the mirror modes are artificially suppressed. In Section 4 we will also make use of the low computational cost of $1 \mathrm{D}$ simulations to test the effect of using values of $m_{i} / m_{e}$ and $\omega_{c, i}^{\text {init }} / s$ that are much larger than the ones used in the $2 \mathrm{D}$ runs. As we will see, we find no significant dependence of the ion acceleration on these parameters.

\footnotetext{
${ }^{12}$ After fitting the low-energy part of $d n_{i} / d \gamma_{i}$ to a thermal MaxwellBoltzmann distribution, we define the nonthermal tail through the condition that $d n_{i} / d \gamma_{i}$ is at least a factor of 2 larger than the expectation for the thermal distribution.
} 

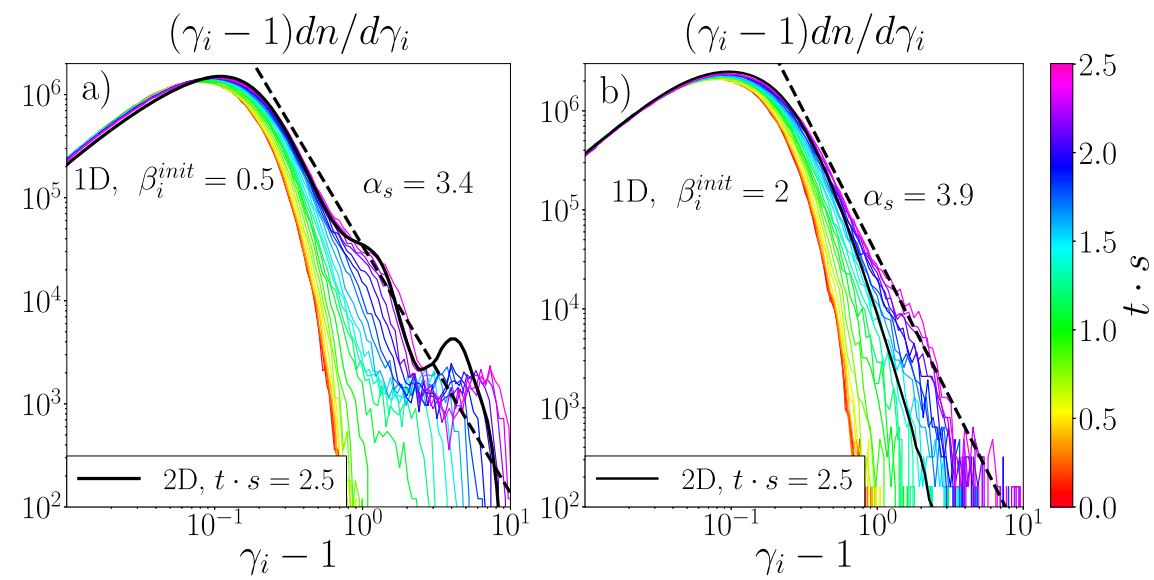

Figure 8. Evolution of the ion spectra for the $1 \mathrm{D}$ runs S1m2b0.5 $\left(m_{i} / m_{e}=2, \beta_{i}^{\text {init }}=0.5\right.$; panel (a)) and S1m2b2 $\left(m_{i} / m_{e}=2, \beta_{i}^{\text {init }}=2\right.$; panel (b)). The color bar indicates the time for each spectrum. The solid black lines show the final spectra $(t \cdot s=2.5)$ of the 2D runs S2m2b0.5 $\left(m_{i} / m_{e}=2, \beta_{i}^{\text {init }}=0.5\right.$; panel (a)) and S2m2b2 $\left(m_{i} / m_{e}=2, \beta_{i}^{\text {init }}=2\right.$; panel (b)), which, apart from the different number of dimensions, assume the same plasma conditions as runs S1m2b0.5 and S1m2b2, respectively.

\section{1D Shearing Simulations}

Since the IC modes propagate mainly parallel to the background magnetic field $\langle\boldsymbol{B}\rangle$, in this section, we study the ion acceleration due to the IC instability by only capturing modes with wavevector $\boldsymbol{k}$ parallel to $\langle\boldsymbol{B}\rangle$. We do so by using the $1 \mathrm{D}$ version of our shear coordinate simulations (Riquelme et al. 2012). Analogous to what happens in 2D and 3D, in our 1D shearing runs, the spatial domain of the simulation evolves with time, following the shearing flow of the plasma. This implies that the domain rotates and stretches so that the resolved $\boldsymbol{k}$ is always parallel to $\langle\boldsymbol{B}\rangle$. A detailed description of our 1D setup is in Appendix A.

In the next section, we show the suitability of the $1 \mathrm{D}$ setup to study problems dominated by modes with $\boldsymbol{k} \| \boldsymbol{B}$ by comparing $1 \mathrm{D}$ runs with $2 \mathrm{D}$ simulations that are dominated by the IC instability. Then, we use the 1D runs to (i) provide further evidence that the IC modes are the essential ingredient for the ion acceleration and (ii) explore the dependence of the ion acceleration on $m_{i} / m_{e}$ and $\omega_{c, i}^{\text {init }} / s$, which will make use of the low computational cost of the $1 \mathrm{D}$ runs.

\section{1. $1 D$ versus $2 D$ Comparison}

We use runs S1m2b0.5 (1D) and S2m2b0.5 (2D) to compare the 1D and 2D results. First, we check whether our 1D and 2D runs give similar ion spectra. Figure 8(a) shows the evolution of the ion spectrum for run S1m2b0.5 (1D) from $t \cdot s=0$ to 2.5. We see that the 1D spectral evolution is very similar to the one of the 2D run S2m2b0.5, shown in Figure 7(a). Indeed, both spectra can be described as a power law of index $\alpha_{s} \approx 3.4$ plus two bumps, with the intermediate-energy bump appearing at $t \cdot s \gtrsim 2$. A more detailed comparison can be made by overplotting the final $(t \cdot s=2.5)$ spectrum for run $\mathrm{S} 2 \mathrm{~m} 2 \mathrm{~b} 0.5$ in Figure 8(a) (solid black line). We see that the two final spectra are very similar, with the main difference being an $\sim$ two times larger maximum energy in the 1D run. This small difference is to some extent expected, due to the presence of mirror modes in the $2 \mathrm{D}$ case. Indeed, in $2 \mathrm{D}$, we have some contributions of mirror modes, which, however, are not conducive to ion acceleration. This explains why the 2D setup leads to somewhat lower energy gains than in 1D.

In terms of the ion pressure anisotropy, Figure 2(a) shows $p_{i}, \|$ (red) and $p_{i}, \perp$ (blue) for runs $\mathrm{S} 1 \mathrm{~m} 2 \mathrm{~b} 0.5$ (1D; dotted) and
$\mathrm{S} 2 \mathrm{~m} 2 \mathrm{~b} 0.5$ (2D; solid). For both $p_{i}, \|$ and $p_{i}, \perp$, the 1D and 2D simulations give essentially the same results. In dashed lines, we show the corresponding double adiabatic behavior (Chew et al. 1956), which is followed quite well by the two simulations until $t \cdot s \approx 1$. With respect to energy conservation, Figure 2(b) shows the volume average $d U_{i} / d t$ (black) and $r \Delta p_{i}$ (green) for the same runs, S1m2b0.5 (1D; dashed) and $\mathrm{S} 2 \mathrm{~m} 2 \mathrm{~b} 0.5$ (2D; solid). Here $d U_{i} / d t$ behaves very similarly in $1 \mathrm{D}$ and $2 \mathrm{D}$, and in the two cases, it corresponds quite well to the heating due to AV.

In order to compare the behavior of the magnetic fluctuations $\delta \boldsymbol{B}$, we use $\delta \boldsymbol{B}_{z}$, since in the 2D runs, this component is mainly produced by the IC modes, and it is essentially not affected by the (subdominant but still present) mirror modes. Figure 9(a) shows a snapshot of $\delta B_{z}$ at $t \cdot s=2$ for the $1 \mathrm{D}$ run $\mathrm{S} 1 \mathrm{~m} 2 \mathrm{~b} 0.5$. We see that in terms of both the dominant wavelength $\left(\approx 30 R_{L, i}^{\text {init }}\right)$ and its amplitude, $\delta B_{z}$ behaves fairly similarly to the 2D case, shown in Figure 3(c). Figures 9(b)-(d) show power spectra of $\delta B_{z}$ at different simulation times for a more detailed comparison. In order to reduce the effects of time variability, we take averages during $1.1<t \cdot s<1.2$, $1.8<t \cdot s<2$, and $2.2<t \cdot s<2.4$, respectively. We see that the 1D and 2D spectra look quite similar. Their main difference consists of a small (by a factor of $\sim 1.5$ ) shift in the peak of the 1D spectra toward longer wavelengths and a factor of $\sim 2$ increase in the peak amplitude.

These differences in the $\delta B_{z}$ spectra can be explained to a large extent by the small differences in the ion energy spectra. Indeed, in Section 5.2 we show that the wavenumber $k$ at the peak of the IC wave spectrum is determined by the resonance condition with the highest-energy ions, with $k \propto 1 / \gamma_{i}$. Thus, since the $1 \mathrm{D}$ runs produce maximum ion energies $\sim$ two times larger than in the 2D case, the wavenumber at the peak should be reduced by a similar factor.

Something similar occurs with the difference in amplitude of $\delta B_{z}$. For relativistic ions interacting resonantly with parallelly propagating waves, the effective scattering frequency $\nu_{\mathrm{eff}, i}$ should scale as (Kulsrud \& Pearce 1969)

$$
\nu_{\mathrm{eff}, i} \propto \frac{\omega_{c, i}}{B_{0}^{2} \gamma_{i}} \frac{d\left(\delta B_{z}^{2}\right)}{d \ln (k)} .
$$

Thus, since the ions in the $1 \mathrm{D}$ and $2 \mathrm{D}$ runs are scattered at roughly the same rate (given their similar evolution of $p_{\|, i}$ and 

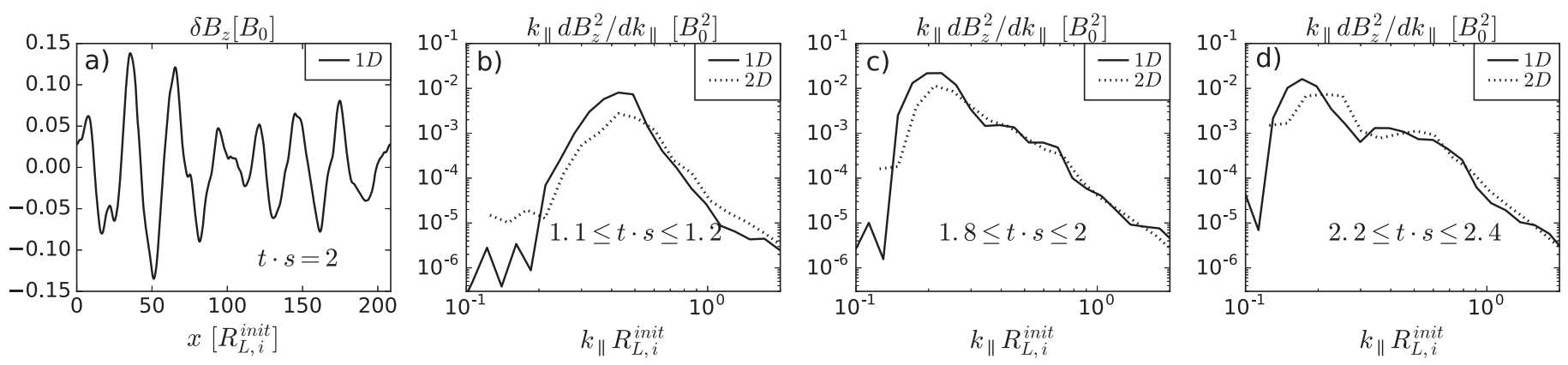

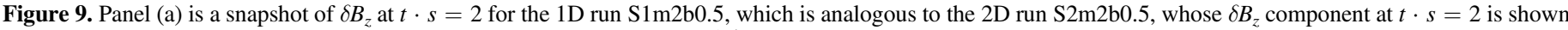

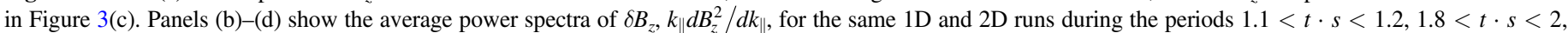
and $2.2<t \cdot s<2.4$, respectively. Here $k_{\|}$denotes the $\boldsymbol{k}$ component parallel to $\boldsymbol{B}$ in the $2 \mathrm{D}$ case, which corresponds to simply $k$ in the $1 \mathrm{D}$ case.
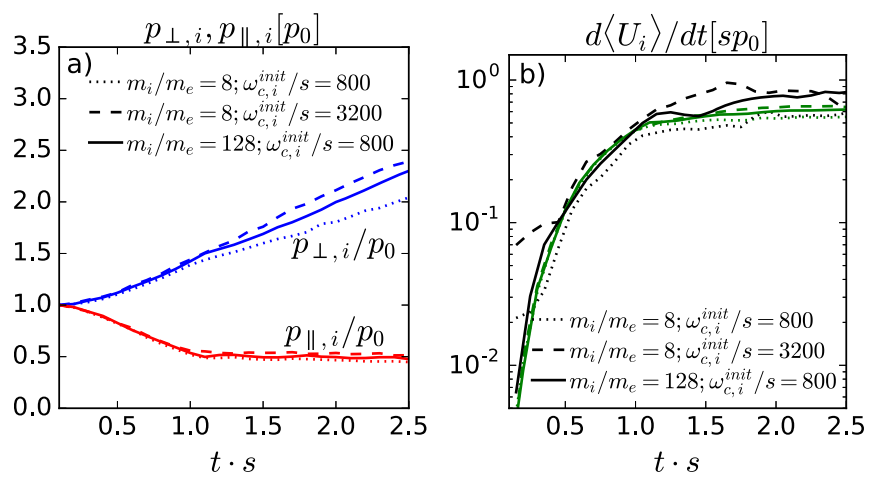

Figure 10. Panel (a): $p_{i}, \|$ (red) and $p_{i}, \perp$ (blue) for $1 \mathrm{D}$ runs $\mathrm{S} 1 \mathrm{~m} 8 \mathrm{~b} 0.5$ (dotted), S1m128b0.5 (solid), and S1m8b0.5d (dashed), all with $\beta_{i}^{\text {init }}=0.5$. Panel (b): volume-averaged $d U_{i} / d t$ (black) and $r \Delta p_{i}$ (green) for the same $1 \mathrm{D}$ runs and using the same line styles. Both $d U_{i} / d t$ and $r \Delta p_{i}$ evolve very similarly in all runs, and in all cases, the ion energy gain reproduces reasonably well the AV prediction $r \Delta p_{i}$ (Equation (1)).

$p_{\perp, i}$, as shown in Figure 2(a)), Equation (2) implies that the peak value of $d B_{z}^{2} k / d k$ in the $1 \mathrm{D}$ case should be roughly two times larger than in the 2D case, which is seen in panels (b)-(d) of Figure 9.

Thus, besides a factor of $\sim 2$ difference in the highest energy of ions (which is likely due to the weak presence of mirror modes in the $2 \mathrm{D}$ runs), the $1 \mathrm{D}$ runs reproduce the $2 \mathrm{D}$ results reasonably well and provide a valuable tool to study the effect of IC waves on ion acceleration. In the next two sections, we use $1 \mathrm{D}$ runs to provide further evidence that the IC modes are indeed the essential ingredient for ion acceleration and explore the dependence of the acceleration on $m_{i} / m_{e}$ and $\omega_{c, i}^{\text {init }} / s$.

\subsection{The Role of IC and Mirror Modes}

Simulations in 1D can be used to further clarify the role of IC and mirror modes in the acceleration of ions. We do this by comparing the ion spectra from a $2 \mathrm{D}$ simulation where the mirror modes dominate $\left(\beta_{i}^{\text {init }}=2\right)$ with an analogous $1 \mathrm{D}$ run where these modes are artificially suppressed. This is done in Figure 8(b), which shows in black the final spectrum of the 2D run $\mathrm{S} 2 \mathrm{~m} 2 \mathrm{~b} 2$, where the mirror modes dominate. In addition, Figure $8(\mathrm{~b})$ shows the ion spectra at different times for the 1D run $\mathrm{S} 1 \mathrm{~m} 2 \mathrm{~b} 2$, where the plasma is under the same conditions as in run $\mathrm{S} 2 \mathrm{~m} 2 \mathrm{~b} 2$. It can be seen that ions tend to be significantly more accelerated in the 1D case, in which the IC modes dominate. This shows that the main ingredient for the acceleration of ions is indeed the scattering by the IC modes,
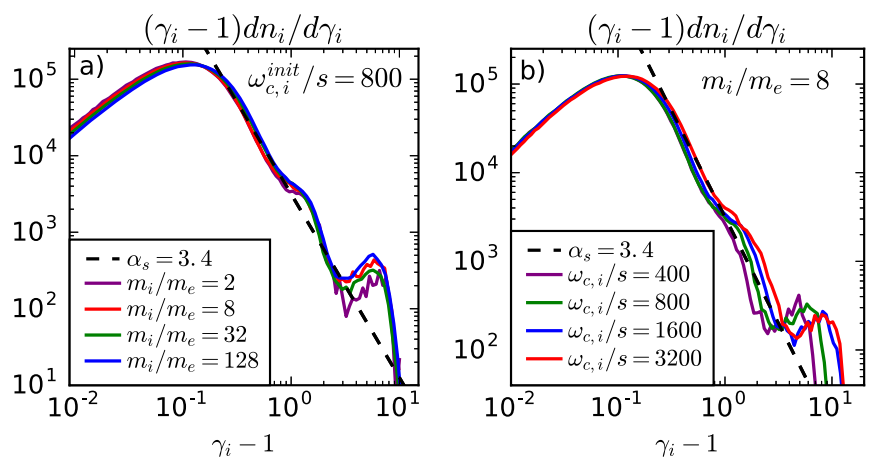

Figure 11. Final ion spectra $(t \cdot s=2.5)$ for 1D simulations with the same parameters $\beta_{i}^{\text {init }}=0.5$ and $k_{B} T_{i}=k_{B} T_{e}=0.05 m_{i} c^{2}$. The runs in panel (a) have $\omega_{c, i}^{\text {init }}=800$ and $m_{i} / m_{e}=2,8,32$, and 128 (respectively, runs $\mathrm{S} 1 \mathrm{~m} 2 \mathrm{~b} 0.5$, $\mathrm{S} 1 \mathrm{~m} 8 \mathrm{~b} 0.5, \mathrm{~S} 1 \mathrm{~m} 32 \mathrm{~b} 0.5$, and $\mathrm{S} 1 \mathrm{~m} 128 \mathrm{~b} 0.5$ in Table 1). The runs in panel (b) have $m_{i} / m_{e}=8$ but with $\omega_{c, i}^{\text {init }} / s=400,800,1600$, and 3200 (runs S1m8b0.5b, $\mathrm{S} 1 \mathrm{~m} 8 \mathrm{~b} 0.5, \mathrm{~S} 1 \mathrm{~m} 8 \mathrm{~b} 0.5 \mathrm{c}$, and $\mathrm{S} 1 \mathrm{~m} 8 \mathrm{~b} 0.5 \mathrm{~d}$, respectively).

with the mirror instability suppressing the acceleration. This suppression is in line with the lack of electric field associated with the mirror modes, as shown in Figure 3. Thus, when the mirror modes dominate, the scattering of ions tends to be elastic, and no acceleration effect should be present.

\section{3. $\mathrm{m}_{\mathrm{i}} / \mathrm{m}_{\mathrm{e}}$ and $\omega_{c, i}^{\text {init }}$ Dependence}

We use 1D simulations to explore the dependence of the ion acceleration by the IC instability on $m_{i} / m_{e}$ and $\omega_{c, i}^{\text {init }} / s$, focusing on the case with $\beta_{i}^{\text {init }}=0.5$. In terms of the evolution of $p_{\perp, i}$ and $p_{\|, i}$, Figure 10(a) shows the cases of runs S1m8b0.5 $\left(\omega_{c, i}^{\text {init }} / s=800, \quad m_{i} / m_{e}=8 ; \quad\right.$ dotted line), S1m128b0.5 $\left(\omega_{c, i}^{\text {init }} / s=800, m_{i} / m_{e}=128 ;\right.$ solid line $)$ and $\mathrm{S} 1 \mathrm{~m} 8 \mathrm{~b} 0.5 \mathrm{~d}$ $\left(\omega_{c, i}^{\text {init }} / s=3200, m_{i} / m_{e}=8\right.$; dashed line). No significant difference can be seen between the different mass ratios and magnetizations. The same thing happens when we look at the ion energy gain. Figure 10(b) shows $d U_{i} / d t$ (black) and $r \Delta p_{i}$ (green) for the same runs. We see that the ion energy gain is fairly independent of $m_{i} / m_{e}$ and $\omega_{c, i}^{\text {init }} / s$, and in all cases, it agrees reasonably well with the heating prediction through AV.

Figure 11(a) shows the final ion spectra $(t \cdot s=2.5)$ for simulations with $m_{i} / m_{e}=2,8,32$, and 128 (runs S1m2b0.5, $\mathrm{S} 1 \mathrm{~m} 8 \mathrm{~b} 0.5, \mathrm{~S} 1 \mathrm{~m} 32 \mathrm{~b} 0.5$, and S1m128b0.5 in Table 1). In all simulations, the ions share the same parameters- $\omega_{c, i}^{\text {init }} / s=$ 800, $\beta_{i}^{\text {init }}=0.5$, and $k_{B} T_{i}=k_{B} T_{e}=0.05 m_{i} c^{2}$ - so the only difference is the value of $m_{i} / m_{e}$. In all cases, the nonthermal 

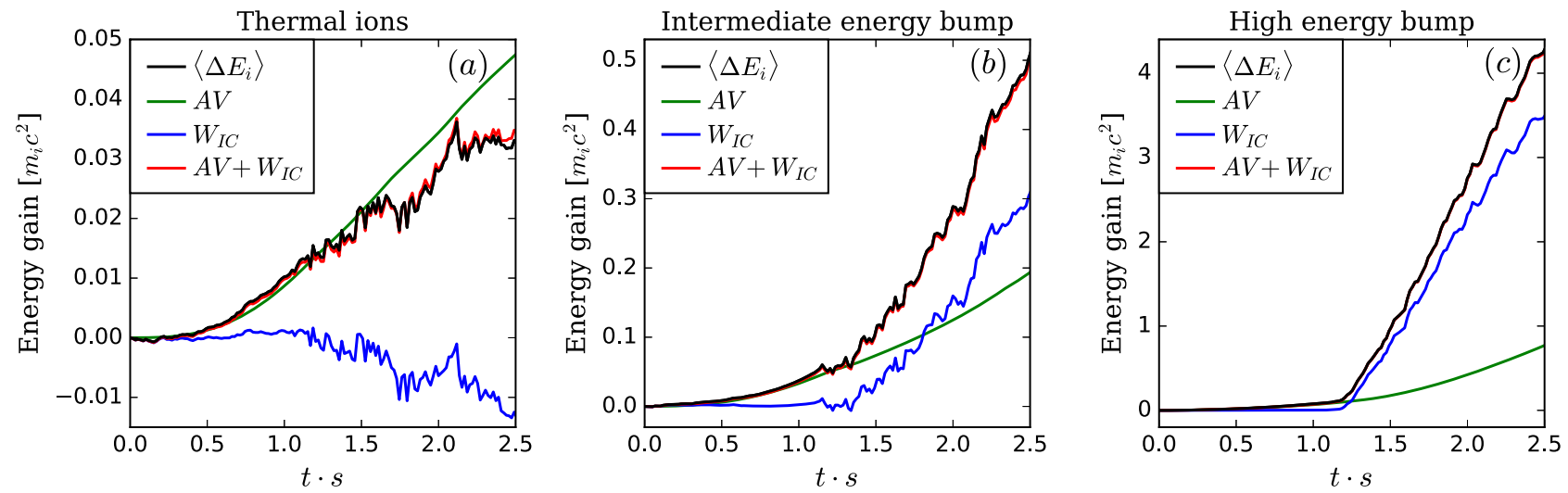

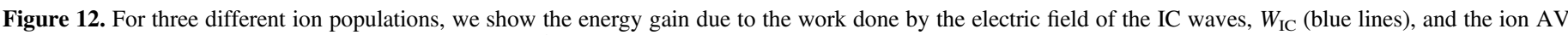

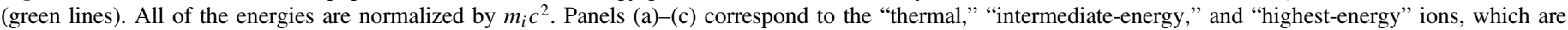

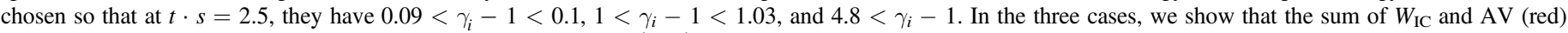
corresponds quite well to the average variation in the ion energy, $\left\langle\Delta E_{i}\right\rangle$ (black).

ion tail can be fairly well described as a power law of spectral index $\alpha_{s} \approx 3.4$ plus two bumps occurring at roughly the same energies. This result shows that, as long as the electrons are somewhat less massive than the ions, their effect on the ion acceleration by IC modes becomes negligible. This is expected given the resonant nature of the interaction between the unstable IC modes and the ions, which requires the modes to be left-handed and circularly polarized (e.g., Gary 1992). This polarization requirement naturally makes it significantly more difficult for the electrons to interact resonantly with the IC modes, even for a mass ratio as small as $m_{i} / m_{e}=2$.

Similarly, Figure 11(b) shows the final spectra for simulations with $m_{i} / m_{e}=8, \beta_{i}^{\text {init }}=0.5$, and $k_{B} T_{i}=k_{B} T_{e}=0.05 m_{i} c^{2}$ but with $\omega_{c, i}^{\text {init }} / s=400,800,1600$, and 3200 (runs $\mathrm{S} 1 \mathrm{~m} 8 \mathrm{~b} 0.5 \mathrm{~b}, \mathrm{~S} 1 \mathrm{~m} 8 \mathrm{~b} 0.5$, S1m8b0.5c, and S1m8b0.5d, respectively). The spectra get slightly harder as $\omega_{c, i}^{\text {init }} / s$ increases, with the difference between them being progressively less significant as $\omega_{c, i}^{\text {init }} / s$ grows. However, in all cases, the tail can be well described as a power law of spectral index $\alpha_{s} \approx 3.4$ plus two bumps.

The independence of the ion acceleration on $\omega_{c, i}^{\text {init }} / s$ can be inferred from the way the effective ion scattering rate $\nu_{\mathrm{eff}, i}$ provided by the IC waves is related to $s$. This scattering rate can be estimated from the evolution of $p_{\|, i}$ in a homogeneous plasma with no heat flux, assuming that $B$ evolves on time and length scales much larger than $\omega_{c, i}^{-1}$ and $R_{L, i}$ (which is the case in our runs). This evolution is given by Equation (1) of Sharma et al. (2007),

$$
\frac{\partial p_{\|, i}}{\partial t}+\nabla \cdot\left(p_{\|, i} \boldsymbol{v}\right)+2 p_{\|, i} \hat{\boldsymbol{b}} \hat{\boldsymbol{b}}: \nabla \boldsymbol{v}=\frac{2}{3} \nu_{\mathrm{eff}, i} \Delta p
$$

where $\boldsymbol{v}$ is the plasma bulk velocity and $\hat{\boldsymbol{b}} \equiv \boldsymbol{B} / \boldsymbol{B}$. In the case of the shearing plasma $(v=-s x \hat{y}), \nabla \cdot\left(p_{\|, i} v\right)=0$ and $\hat{\boldsymbol{b}} \hat{\boldsymbol{b}}: \nabla \boldsymbol{v}=\hat{b}_{x} \hat{b}_{y} s$. Figure 10(a) shows that, after the saturation of the IC modes, $p_{\|, i}$ changes at a rate much smaller than $s$, so we can approximate $\partial p_{\|, i} / \partial t \approx 0$. Additionally, $\hat{b}_{x} \hat{b}_{y}$ ranges between 0.5 at $t \cdot s=1$ and 0.34 at $t \cdot s=2.5$. Thus, we simply assume $\hat{b}_{x} \hat{b}_{y} \approx 1 / 2$, and Equation (3) becomes

$$
\nu_{\mathrm{eff}, i} \approx \frac{3}{2} s\left(p_{\|, i} / \Delta p_{i}\right)
$$

By comparing the evolution of $p_{\perp, i}$ and $p_{\|, i}$ for runs $\mathrm{S} 1 \mathrm{~m} 8 \mathrm{~b} 0.8$ $\left(\omega_{c, i}^{\text {init }} / s=800\right.$; dotted line $)$ and S $1 \mathrm{~m} 8 \mathrm{~b} 0.8 \mathrm{~d}\left(\omega_{c, i}^{\text {init }} / s=3200\right.$; dashed line), Figure 10(a) shows that the factor $p_{\|, i} / \Delta p_{i}$ is fairly independent of $\omega_{c, i}^{\text {init }} / s$. Thus, Equation (4) implies that $\nu_{\mathrm{eff}, i} \propto s$.

This proportionality between $\nu_{\text {eff }, i}$ and $s$ means that the average number of scatterings experienced by the ions after $t=2.5 \mathrm{~s}^{-1}$ (at the end of the simulations) should be about the same in all runs. This property, if the IC modes' properties are the same in all simulations (as occurs with the runs shown in Figure 11(b)), should make the accelerating effect of the IC modes independent of $\omega_{c, i}^{\text {init }} / s$.

Our 1D simulations, therefore, show that the ion acceleration by IC modes is fairly independent of $m_{i} / m_{e}$ and $\omega_{c, i}^{\text {init }} / s$. In the next section, we use $1 \mathrm{D}$ and $2 \mathrm{D}$ simulations to describe in further detail the way that ions of different energy get accelerated, emphasizing the role played by their resonant interaction with the IC waves.

\section{The Acceleration Mechanism}

The two possible sources of energy for the ions in our simulations are the energy gains due to AV (Equation (1)) and the work done by the electric field associated with the IC waves (considering that the electric field associated with the mirror modes is negligible). In Section 5.1 we identify the contributions of each of these energy sources to producing the nonthermal ion spectra.

\subsection{IC Work versus AV}

Figure 12 shows the different contributions to the energy gain of three ion populations from the $2 \mathrm{D}$ run $\mathrm{S} 2 \mathrm{~m} 2 \mathrm{~b} 0.5$, separated according to their final energy at $t \cdot s=2.5$. These populations are as follows.

1. The "thermal ions," corresponding to ions in the bulk of the ion distribution, with their energy gain plotted in Figure 12(a). These ions are chosen so that at $t \cdot s=2.5$, their Lorentz factors satisfy $0.09<\gamma_{i}-1<0.1$ (marked by the vertical red line in Figure 7(a)).

2. The ions in the "intermediate-energy" bump of the tail (shown in Figure 12(b)). These ions have Lorentz factors 


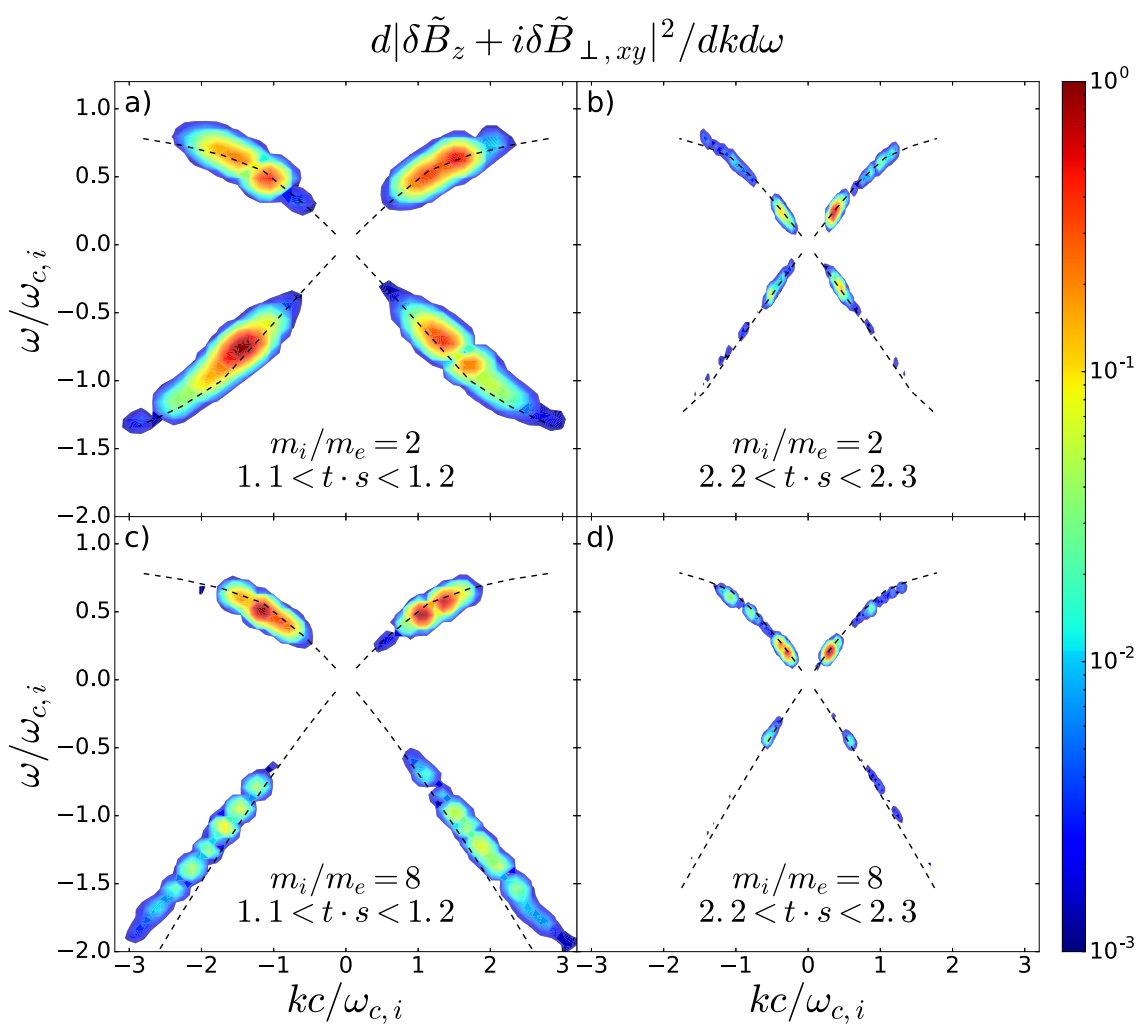

Figure 13. The four panels show $\left|\delta \tilde{B}_{z}(\omega, k)+i \delta \tilde{B}_{\perp, x y}(\omega, k)\right|^{2}$ for different $1 \mathrm{D}$ simulations and time intervals, where $\delta \tilde{B}_{z}(\omega, k)$ and $\delta \tilde{B}_{\perp, x y}(\omega, k)$ are the FTs in time and space of the two mutually perpendicular components of $\delta \boldsymbol{B}, \delta B_{z}$ and $\delta B_{\perp, x y}$ (both perpendicular to $\left.\langle\boldsymbol{B}\rangle\right)$. The combination $\delta \tilde{B}_{z}(\omega, k)+i \delta \tilde{B}_{\perp, x y}(\omega, k)$ makes the contributions to $\delta \boldsymbol{B}$ of the circularly polarized IC waves (left-handed) and whistler waves (right-handed) appear at $\omega>0$ and $<0$, respectively. Panels (a) and (b) correspond to run $\mathrm{S} 1 \mathrm{~m} 2 \mathrm{~b} 0.5\left(\beta_{i}^{\text {init }}=0.5, m_{i} / m_{e}=2, \omega_{c, i}^{\text {init }} / s=800\right)$ at $1.1<t \cdot s<1.2$ and $2.2<t \cdot s<2.3$, respectively. Panels (c) and (d) correspond to run $\mathrm{S} 1 \mathrm{~m} 8 \mathrm{~b} 0.5\left(\beta_{i}^{\text {init }}=0.5, m_{i} / m_{e}=8, \omega_{c, i}^{\text {init }} / s=800\right)$ also at $1.1<t \cdot s<1.2$ and $2.2<t \cdot s<2.3$, respectively. Here $\omega$ is normalized to the "instantaneous" cyclotron frequency $\omega_{c, i}\left(\equiv \omega_{c, i}^{\text {init }} B / B_{0}\right)$, while $k$ is normalized to $\omega_{c, i} / c$. With dashed lines, we show the theoretical IC and whistler dispersion relations $\omega_{\text {theo }}(k)$ obtained with the linear Vlasov solver NHDS (Verscharen \& Chandran 2018).

in the range $1<\gamma_{i}-1<1.03$ at $t \cdot s=2.5$ (marked by the vertical dark green line in Figure 7(a)).

3. The ions in the "high-energy" bump of the tail (shown in Figure 12(c)). This population corresponds to the highestenergy ions, defined by $\gamma_{i}-1>4.8$ at $t \cdot s=2.5$ (marked by the gray region in Figure 7(a)).

For each of these populations, we plot the following contributions to their energy gain:

1. the work done by the electric field of the IC waves, $W_{\mathrm{IC}}$, which is shown by the blue lines of Figure 12, and

2. the energy gain by $\mathrm{AV}$, shown by the green lines in Figure $12 .{ }^{13}$

The blue line in Figure 12(a) shows that the energy given by the electric field of the IC waves to the thermal ions, $W_{\mathrm{IC}}$, is negative. This implies that the scattering process, on average, subtracts energy from the thermal ions and transfers it to the waves. The total gain in energy of the thermal ions is still positive and dominated by viscous heating. On the other hand, Figure 12(b) shows that the work done by the IC waves on the ions of the intermediate-energy bump is positive and larger than the heating by $\mathrm{AV}$, which means that these ions are mainly energized by the scattering caused by the IC waves. This energization occurs mainly after $t \cdot s \approx 2$, which is consistent

\footnotetext{
${ }^{13}$ For each ion population, this energy gain is calculated as the integral in time of the rate of energy gain due to viscosity, $\int d t r \Delta p_{i}$ (see Equation (1)), where $\Delta p_{i}$ is calculated using only the ions of each population.
}

with the late-time appearance of the intermediate-energy bump, as shown by the time evolution of the ion spectrum depicted in Figure 7(a). Finally, Figure 12(c) shows that $W_{\text {IC }}$ is about three times larger than $\mathrm{AV}$, implying that the IC acceleration for the highest-energy ions is even larger than for the intermediateenergy ions.

In Figures 12(a)-(c), we also plot the sum of $\mathrm{AV}$ and $W_{\mathrm{IC}}$ (red lines) and the average change in energy of the three ion populations, $\left\langle\Delta E_{i}\right\rangle$ (black lines). We see that these two quantities are essentially the same for the three populations, implying that the energy gain due to AV and the electric field of the IC modes accounts quite well for the total ion energy evolution in the three populations.

\subsection{Resonance with IC Waves}

In Section 5.1 we show that a nonthermal ion tail is produced by the scattering of ions off IC waves, which, in turn, obtain their energy from the pressure anisotropy of the thermal ions. This implies that this acceleration mechanism requires the resonance condition between ions and IC waves to be satisfied by both the thermal and nonthermal ions. The resonance condition is

$$
\frac{\omega}{k}-v_{\|}=\frac{\omega_{c, i}}{\gamma_{i} k}
$$

where $\omega$ and $k$ are the real part of the frequency and the wavenumber of the modes, $v_{\|}$is the ion velocity parallel to $\boldsymbol{B}$, 
and $\omega_{c, i}\left(\equiv e B / m_{i} \mathrm{c}\right)$ is the nonrelativistic cyclotron frequency of the ions.

In order to check that this resonance condition is satisfied by ions of all energies, in Figures 13(a) and (b), we measure the ratio $\omega / k$ for the IC waves at two different time intervals for the 1D run $\mathrm{S} 1 \mathrm{~m} 2 \mathrm{~b} 0.5\left(\beta_{i}^{\text {init }}=0.5, m_{i} / m_{e}=2, \omega_{c, i}^{\text {init }}=800\right)$. We do this by plotting $\left|\delta \tilde{B}_{z}(\omega, k)+i \delta \tilde{B}_{\perp, x y}(\omega, k)\right|^{2}$, where $\delta \boldsymbol{B}_{z}(\omega, k)$ and $\delta \boldsymbol{B}_{\perp x y}$ correspond to two mutually perpendicular components of $\delta \boldsymbol{B}$ that are also perpendicular to $\langle\boldsymbol{B}\rangle$ (see caption of Figure 5), the tilde $(\sim)$ denotes the time and space FT of a quantity, and $i=\sqrt{-1}$. Thus, the combination $\delta \tilde{B}_{z}(\omega, k)+i \delta \tilde{B}_{\perp, x y}(\omega, k)$ allows us to separate the contributions to $\delta \boldsymbol{B}$ provided by IC waves (lefthanded, circularly polarized) and whistler waves (right-handed, circularly polarized), with the latter expected to be destabilized by the pressure anisotropy of electrons (Gary \& Wang 1996). In the case of run $\mathrm{S} 1 \mathrm{~m} 2 \mathrm{~b} 0.5$, the IC waves contribute to $\left|\delta \tilde{B}_{z}(\omega, k)+i \delta \tilde{B}_{\perp, x y}(\omega, k)\right|$ only for $\omega>0$, while the whistler wave contribution appears for $\omega<0$. This way, calculating $\left|\delta \tilde{B}_{z}(\omega, k)+i \delta \tilde{B}_{\perp, x y}(\omega, k)\right|^{2}$ allows us to separate the IC and whistler contributions to $\delta \boldsymbol{B}$ and estimate $\omega(k)$ for these two modes.

Figure 13(a) corresponds to the time interval $1.1<t \cdot s<$ 1.2 of run $\mathrm{S} 1 \mathrm{~m} 2 \mathrm{~b} 0.5$. The IC modes have a phase velocity of $\omega / k \approx 0.5 c$. Additionally, we measure the rms ion velocity parallel to $\boldsymbol{B}$ at $1.1<t \cdot s<1.2$, which is $v_{\|}^{\text {rms }} \approx 0.16 c{ }^{14}$ implying that, to a good approximation, we can neglect the $v_{\|}$ term on the left-hand side of Equation (5). The resonance condition at $1.1<t \cdot s<1.2$ can thus be written as

$$
\gamma_{i}\left(k c / \omega_{c, i}\right) \approx 2 \text {. }
$$

At $1.1<t \cdot s<1.2, \gamma_{i}$ is in the range $1<\gamma_{i} \lesssim 2$ (see Figure 8(a)), implying that most of the power of the IC modes should be in the range $1 \lesssim k c / \omega_{c, i} \lesssim 2$, which coincides well with the range of $k$ in which most of the IC power is observed in Figure 13(a).

Analogously, Figure 13(b) shows $\left|\delta B_{z}(\omega, k)+i \delta B_{\perp, x y}(\omega, k)\right|^{2}$ for the same simulation but in the time range $2.2<t \cdot s<2.3$. In this case, Figure $13(\mathrm{~b})$ shows that $\omega / k \approx 0.7 c$. Thus, making a similar analysis as in the case $1.1<t \cdot s<1.2$, we obtain ${ }^{15}$

$$
\gamma_{i}\left(k c / \omega_{c, i}\right) \approx 1.5 \text {. }
$$

Since in this time interval, $1<\gamma_{i} \lesssim 8$, we obtain that most of the power of the IC modes should be in the range $0.2 \lesssim k c / \omega_{c, i} \lesssim 1.5$. This interval coincides reasonably well with the range of $k$ where the most power is concentrated in Figure 13(b). Notice that this power appears to be enhanced in two intervals of $k$. The high- $k$ interval corresponds to $0.7 \lesssim k c / \omega_{c, i} \lesssim 1.5$, which, according to Equation (7), implies resonance with ions with $1<\gamma_{i} \lesssim 2$. Remarkably, this is a range of $\gamma_{i}$ with abundant IC scattering at $2.2 \lesssim t \cdot s \lesssim 2.3$, as shown by the rapid formation of the "intermediate-energy" bump, which mainly occurs at $2 \lesssim t \cdot s \lesssim 2.5$. The low- $k$ enhancement occurs for $0.2 \lesssim k c / \omega_{c, i} \lesssim 0.5$, which, according to Equation (7), corresponds to $3 \lesssim \gamma_{i} \lesssim 8$. This $\gamma_{i}$ interval

\footnotetext{
${ }^{14}$ This can be estimated by the factor of $\sim 2$ decrease in $p_{i}, \|$ seen in Figure 2(a) and considering that initially, $k_{B} T_{i} / m_{i} c^{2}=0.05$.

${ }^{15}$ Here we also assume $\omega / k(\approx 0.7 c) \gg v_{\|}$, which allows neglecting $v_{\|}$in Equation (5). This is a reasonable approximation, considering that at $2.2<t \cdot s<2.3, v_{\|}$(measured directly from the simulation) is always smaller than $\sim 0.4 c$, even considering the highest-energy particles in the nonthermal tail.
}

coincides well with the "high-energy" bump shown in Figure 8 (a) at $t \cdot s \sim 2.2-2.3$ and is also consistent with the rapid increase in energy of this bump.

Thus, we have shown that the range of $k$ in which the amplitude of the IC modes is significant is consistent with the resonance condition occurring for both the thermal and nonthermal ions in the tail. Figures 13(c) and (d) show the same quantities as Figures 13(a) and (b) but for simulation $\mathrm{S} 1 \mathrm{~m} 8 \mathrm{~b} 0.5$, where the ions are under the same conditions as in run $\mathrm{S} 1 \mathrm{~m} 2 \mathrm{~b} 0.5$, but with $m_{i} / m_{e}=8$ instead of $m_{i} / m_{e}=2$. The quantity $\left|\delta \tilde{B}_{z}(\omega, k)+i \delta \tilde{B}_{\perp, x y}(\omega, k)\right|^{2}$ essentially preserves the same properties for the IC modes $(\omega>0)$. The fact that $\omega(k)$ of the IC modes is fairly independent of $m_{i} / m_{e}$ is consistent with the near-independence of the ion acceleration process on $m_{i} / m_{e}$. The whistler modes, on the other hand, do significantly change their properties as $m_{i} / m_{e}$ is increased, for instance, by getting weaker (which is consistent with the discussion in footnote 14) and increasing their frequency. This is expected, since quantities like $\omega_{c, e} / \omega_{p, e}, \omega_{c, e} / s$, and $k_{B} T_{e} / m_{e} c^{2}$ do change when varying $m_{i} / m_{e}$.

Finally, the four panels in Figure 13 also include IC and whistler theoretical dispersion relation calculations, $\omega_{\text {theo }}(k)$ (dashed lines), obtained with the linear Vlasov solver NHDS (Verscharen \& Chandran 2018). These calculations assume the ion and electron conditions obtained in the simulations in terms of their temperatures, pressure anisotropies, mass ratio, and Alfvén velocity. However, they do not consider departures from Maxwell-Boltzmann distributions or relativistic effects. In the four panels, $\omega_{\text {theo }}(k)$ well reproduces the behavior of $\left|\delta \tilde{B}_{z}(\omega, k)+i \delta \tilde{B}_{\perp, x y}(\omega, k)\right|^{2}$ for the case of the IC modes, which shows that the phase (and group) velocity of the IC waves obtained from the simulations is not significantly affected by the nonthermal or relativistic effects in the ion velocity distribution. Most discrepancies occur for whistler waves when $m_{i} / m_{e}=8$. In this case, the discrepancy is most likely due to the electrons being significantly relativistic (Lorentz factor $\gamma_{e} \gg 1$ ), which is a regime strictly not captured by NHDS.

\section{Comparing Shear versus Compression}

The ion acceleration presented in this paper occurs during the nonlinear, saturated stage of the IC instability. This stage is reached through the continuous amplification of a background magnetic field $\langle\boldsymbol{B}\rangle$, which in previous sections has been driven by an imposed shear plasma motion. In this section, we show that, as long as the ion conditions are similar, the specific process that amplifies $\langle\boldsymbol{B}\rangle$ does not play an important role in the acceleration. We do this by running 1D simulations in which $\langle\boldsymbol{B}\rangle$ grows due to plasma compression. The simulation setup is the same as in Sironi \& Narayan (2015). The plasma is compressed along the $y$ and $z$-axes, with $\langle\boldsymbol{B}\rangle$ pointing along $x$. In this setup, $\langle\boldsymbol{B}\rangle$ is amplified due to magnetic flux conservation (see Figure 1 of Sironi \& Narayan 2015). In this situation, the background field grows as $|\langle\boldsymbol{B}\rangle|=B_{0}(1+q t)^{2}$, where $q$ is a constant that roughly corresponds to the compression rate of the plasma. These 1D simulations only capture modes with $\boldsymbol{k} \|\langle\boldsymbol{B}\rangle$, which means that the mirror modes are artificially suppressed.

The compression runs use the same initial plasma parameters as the shearing runs that show significant ion acceleration $-\beta_{i}^{\text {init }}=0.5, k_{B} T_{i} / m_{i} c^{2}=0.05$, and $T_{i}=T_{e}$-which allows a direct comparison between the two setups. Figure 14(a) shows the evolution of $T_{\perp, i}$ (solid blue) and $T_{\|, i}$ (solid red) for run C1m8b0.5a, which uses $m_{i} / m_{e}=8$ and $\omega_{c, i}^{\text {init }} / q=1600$ 

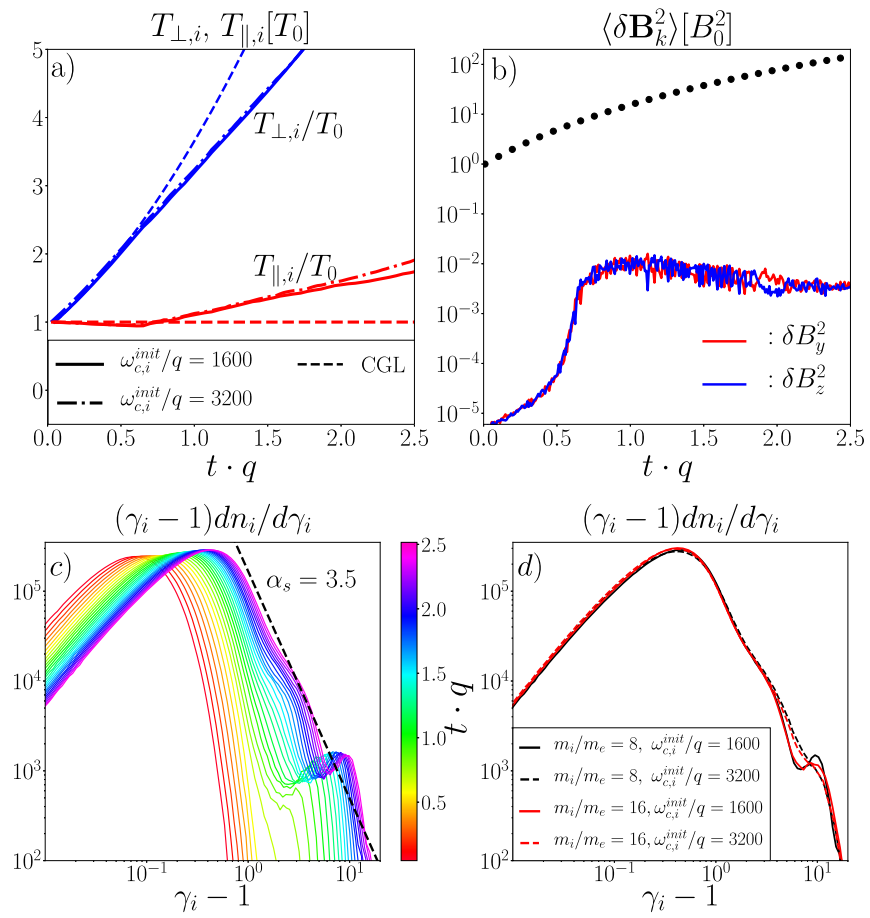

Figure 14. Panel (a) shows the evolution of $T_{\perp, i}$ (blue) and $T_{\|, i}$ (red) for compressing runs $\mathrm{C} 1 \mathrm{~m} 8 \mathrm{~b} 0.5 \mathrm{a}\left(m_{i} / m_{e}=8\right.$ and $\omega_{c, i}^{\text {init }} / q=1600$; solid $)$ and C1m8b0.5b $\left(m_{i} / m_{e}=8\right.$ and $\omega_{c, i}^{\text {init }} / q=3200$; dashed-dotted $)$, both with $\beta_{i}^{\text {init }}=0.5$ and $k_{B} T_{i}=k_{B} T_{e}=0.05 m_{i} c^{2}$. The double adiabatic predictions are shown with dashed lines (Chew et al. 1956). Panel (b) shows $\delta B_{y}^{2}$ (red) and $\delta B_{z}^{2}$ (blue) normalized to $B_{0}^{2}$. The dotted line shows $|\langle\boldsymbol{B}\rangle|^{2}$. Panel (c) shows the evolution of the ion spectrum of run $\mathrm{C} 1 \mathrm{~m} 8 \mathrm{~b} 0.5 \mathrm{a}$. Panel (d) shows a comparison of the spectra at $t \cdot q=2.5$ for runs with $m_{i} / m_{e}=8$ and $\omega_{c, i}^{\text {init }} / q=1600$ (C1m8b0.5a; solid black), $m_{i} / m_{e}=8$ and $\omega_{c, i}^{\text {init }} / q=3200$ (C1m8b0.5b; dashed black), $m_{i} / m_{e}=16$ and $\omega_{c, i}^{\text {init }} / q=1600$ (C1m16b0.5a; solid red), and $m_{i} / m_{e}=16$ and $\omega_{c, i}^{\text {init }} / q=3200(\mathrm{C} 1 \mathrm{~m} 16 \mathrm{~b} 0.5 \mathrm{~b}$; dashed red).

$\left(k_{B} T_{\perp, i}=p_{\perp, i} / n_{i}\right.$ and $k_{B} T_{\|, i}=p_{\|, i} / n_{i}$, where $n_{i}$ is the ion density). ${ }^{16}$ Both temperatures initially follow the double adiabatic evolution reasonably well, which is marked with the dashed blue and red lines, respectively (Chew et al. 1956). ${ }^{17}$ The adiabatic evolution breaks at $t \cdot q \approx 0.6$, after the exponential growth of the IC modes begins. This exponential growth and subsequent saturation can be seen in Figure 14(b), which shows the $\delta B_{y}^{2}$ and $\delta B_{z}^{2}$ components of run $C 1 \mathrm{~m} 8 \mathrm{~b} 0.5 \mathrm{a}$, which evolve quite similarly to the $\delta B_{\perp, x y}$ and $\delta B_{z}^{2}$ fluctuations of analog shearing runs (see, e.g., the cases of runs $\mathrm{S} 2 \mathrm{~m} 2 \mathrm{~b} 0.5$ and $\mathrm{S} 2 \mathrm{~m} 10 \mathrm{~b} 0.5$ in Figure 5(a)).

The evolution of the ion spectrum of run $\mathrm{C} 1 \mathrm{~m} 8 \mathrm{~b} 0.5 \mathrm{a}$ is shown in Figure 14(c). We see a remarkable similarity with the spectral evolution of the shearing runs with $\beta_{i}^{\text {init }}=0.5$, shown in Figures 7(a) and 8(a). Indeed, also in the compressing case, there is a growth of a nonthermal tail that, by $t \cdot q=2.5$, can be described as a power law of index $\alpha \approx 3.5$ plus two bumps.

We also test the dependence of this acceleration on both $m_{i} / m_{e}$ and ion magnetization $\omega_{c, i}^{\text {init }} / q$. Figure 14(d) shows a comparison between cases with $m_{i} / m_{e}=8$ and 16 (black and red, respectively) and $\omega_{c, i} / q=1600$ and 3200 (solid and

\footnotetext{
${ }^{16}$ We show $T_{\perp, i}$ and $T_{\|, i}$ instead of $p_{\perp, i}$ and $p_{\|, i}$ in order to disentangle variations in $n_{i}$ through the compression from the variation through double adiabatic and instability-induced effects.

17 The small initial discrepancies $(t \cdot s \leqslant 0.6)$ between the double adiabatic predictions and $T_{\|, i}$ and $T_{\perp, i}$ are because these predictions assume that the ions are nonrelativistic, which is mildly broken for our $k_{B} T_{i} / m_{i} c^{2} \sim 0.1$ ions.
}

dashed, respectively), which share the same initial parameters: $\beta_{i}^{\text {init }}=0.5, k_{B} T_{i} / m_{i} c^{2}=0.05$, and $T_{i}=T_{e}$. We see essentially no difference between the runs with different values of $m_{i} / m_{e}$ and only a slight hardening of the tail as $\omega_{c, i} / q$ increases, in agreement with the shearing results of Section 4.3.

These results essentially reproduce our shearing runs, both in terms of the significance of the ion acceleration in the regime, $\beta_{i}^{\text {init }}=0.5, k_{B} T_{i} / m_{i} c^{2}=0.05$, and $T_{i}=T_{e}$, and in terms of the almost independence on $m_{i} / m_{e}$ and $\omega_{c, i} / q$.

As in the shearing case, the independence on $m_{i} / m_{e}$ can be understood as due to the resonant nature of the ion-IC interaction. The independence on $\omega_{c, i}^{\text {init }} / q$ implies, similarly to the shearing case, that the effective ion scattering rate $\nu_{\mathrm{eff}, i}$ has to be $\propto q$. This can indeed be inferred by applying Equation (3) to the compressing runs. In this case, $\hat{\boldsymbol{b}} \hat{\boldsymbol{b}}: \nabla \boldsymbol{v}=0$, and thus (using the continuity relation $\partial n_{i} / \partial t=-n_{i} \nabla \cdot v$ ) $n_{i} \partial T_{\|, i} / \partial t=(2 / 3) \nu_{\text {eff }, i} \Delta p_{i}$. However, Figure 14(a) shows that, after the saturation of the IC waves, $\partial T_{\|, i} / \partial t \sim(q / 2) T_{\|, i}$, which suggests

$$
\nu_{\mathrm{eff}, i} \approx \frac{3}{4} q\left(p_{\|, i} / \Delta p_{i}\right) .
$$

The ratio $p_{\|, i} / \Delta p_{i}$ evolves fairly independently of $\omega_{c, i}^{\text {init }} / q$, as can be seen from Figure 14(a), which shows $T_{\perp, i}$ and $T_{\|, i}$ for runs $\mathrm{C} 1 \mathrm{~m} 8 \mathrm{~b} 0.5 \mathrm{a}\left(\omega_{c, i}^{\text {init }} / q=1600\right.$; solid line) and $\mathrm{C} 1 \mathrm{~m} 8 \mathrm{~b} 0.5 \mathrm{~b}$ ( $\omega_{c, i}^{\text {init }} / q=3200$; dotted-dashed line). Therefore, Equation (8) implies that $\nu_{\text {eff }, i} \propto q$.

The similarity between Equations (4) and (8) is consistent with the ion spectra being similar in the two setups when comparing spectra at equal values of $t s$ and $t q$. Indeed, considering that in the shearing and compressing cases, $p_{\|, i} / \Delta p_{i} \sim 1 / 2$ during most of the saturated IC regime (see Figures 2(a) and 14(a)), at equal values of $t s$ and $t q$, the ions must have experienced a similar number of effective scatterings. Thus, if the properties of the IC modes are comparable, their acceleration effects by the end of the shearing and compressing simulations should also be comparable. ${ }^{18}$

\section{Summary and Conclusions}

Our 1D and 2D PIC plasma simulations show that ions can be stochastically accelerated by the inelastic scattering provided by the IC instability. This acceleration occurs in the nonlinear, saturated state of the instability, which is reached due to a permanent amplification of the background magnetic field $\langle\boldsymbol{B}\rangle$.

In the regime in which initially, $k_{B} T_{i}=k_{B} T_{e}=0.05 m_{i} c^{2}$, we show that the IC ion acceleration is significant if $\beta_{i}^{\text {init }} \lesssim 1$. This is demonstrated by comparing $2 \mathrm{D}$ simulations with $\beta_{i}^{\text {init }}=0.5$ and 2 . When $\beta_{i}^{\text {init }}=0.5$, the ion scattering is dominated by the IC instability, which produces a nonthermal tail in the ion energy spectrum. After $\boldsymbol{B}$ is amplified by a factor of $\sim 2.7$, the tail can be approximately described as a power law of index 23.4 plus two nonthermal bumps. The maximum ion Lorentz

\footnotetext{
18 Notice, however, that, although the initial ion conditions in the shearing and compressing runs are the same, the final conditions are somewhat different. For instance, in the shearing runs with $\beta_{i}^{\text {init }}=0.5$, the final value of the parallel ion $\beta$ is $\beta_{\|, i}^{\text {final }} \sim 0.025$ (considering the evolution of $p_{\|, i}$ seen in Figure 2 (a) and the expected evolution of $B$ ), while in the analogous compressing runs, $\beta_{\|, i}^{\text {final }} \sim 0.1$ (considering the evolution of $T_{\|, i}$ seen in Figure 14(a) and the expected evolutions of $n_{i}$ and $B$ ). This, plus the different factors on the right-hand sides of Equations (4) and (8), implies that the final ion spectra in these two setups should be similar but not necessarily the same.
} 
factor at that time is $\gamma_{i} \sim 10$, but it continues to grow at the end of the simulation. Also, the tail accounts for $2 \%-3 \%$ of the ions and $\sim 18 \%$ of their kinetic energy. On the other hand, when $\beta_{i}^{\text {init }}=2$, the ion scattering is dominated by the mirror instability (the IC modes are subdominant), and the acceleration is significantly suppressed.

In the IC-dominated regime, as the ion scattering increases the energy of the ions of the tail, it reduces the energy of the ions in the thermal part of the spectrum (see Figure 12). This is consistent with the IC modes being driven unstable mainly by the pressure anisotropy of the thermal ions. This way, the role of the IC modes is to absorb part of the energy of the thermal ions and give it to the nonthermal ions. This process is very similar to the stochastic acceleration of electrons by the whistler instability found by Riquelme et al. (2017). The efficiency of the ion acceleration, therefore, relies on the IC modes being able to provide resonant scattering to both thermal and nonthermal ions. We analyzed the consistency of this scenario by calculating the $k$ numbers and phase velocities of the dominant IC modes, showing that they can resonate with ions of all energies (see discussion in Section 5.2).

Given that our simulations cannot use realistic values of $m_{i} / m_{e}$ and $\omega_{c, i}^{\text {init }} / s$, one important aspect of our study is to ensure that these parameters do not affect the acceleration. Thus, we first ensure that the dominance of the IC modes for $\beta_{i}^{\text {init }}=0.5$ does not depend on $m_{i} / m_{e}$ and $\omega_{c, i}^{\text {init }} / s$. This was done by comparing 2D simulations with $m_{i} / m_{e}=2$ and 10 (see Section 3.1) and also using theoretical, linear dispersion relation calculations to determine the pressure anisotropy needed for the growth of the IC and mirror instabilities in astrophysically realistic conditions (see Appendix B). Both analyses show that, in realistic astrophysical plasmas, the IC instability dominates in the regime $\beta_{i}^{\text {init }} \lesssim 1$, at least for the case of $T_{e}=T_{i}$ explored here.

Then, using the fact that the dominant IC wavevectors $\boldsymbol{k}$ satisfy $\boldsymbol{k} \|\langle\boldsymbol{B}\rangle$, we use computationally cheaper 1D shear simulations to test the ion acceleration using a significantly larger range of values for $m_{i} / m_{e}$ and $\omega_{c, i}^{\text {init }} / s$. While $m_{i} / m_{e}$ almost does not affect the acceleration, increasing $\omega_{c, i}^{\text {init }} / s$ only produces a slight hardening of the nonthermal tail (see Section 4.3). This almost complete independence of the acceleration on $\omega_{c, i}^{\text {init }} / s$ is consistent with the effective ion scattering rate $\nu_{\text {eff, } i}$ being proportional to $s$. This condition is indeed needed in order to have the continuous driving of the ion pressure anisotropy being nearly compensated by the pitch-angle scattering.

In order to assess the importance of the specific large-scale mechanism that amplifies the background magnetic field, we also ran compressing-box PIC simulations like in Sironi \& Narayan (2015). We find essentially no difference in the ion acceleration efficiency between the shearing and compressing cases (see Section 6).

Our work is valid in a subrelativistic regime in which initially, $k_{B} T_{i}=k_{B} T_{e}=0.05 m_{i} c^{2}$. This regime can be relevant in the inner region of low-luminosity accretion disks around black holes (where the collisionless plasma condition is expected). In these systems, the condition $\beta_{i}^{\text {init }} \lesssim 1$ required for the acceleration is most likely satisfied in the coronal region of the disks (e.g., Chael et al. 2018).

Nevertheless, assessing the importance of the presented acceleration mechanism in these and other astrophysical systems requires a more complete understanding of its dependence on plasma parameters, as well as clarifying the importance of possible long-term evolution effects. Indeed, in this work, we focus on a single value of $k_{B} T_{i}$ and use $T_{e} / T_{i}=1$. However, varying these parameters may affect the IC physics and, therefore, the efficiency of the ion acceleration. For instance, having $T_{e} / T_{i} \ll 1$ may significantly increase the values of $\beta_{i}^{\text {init }}$ for which the IC instability dominates (Sironi 2015; Sironi \& Narayan 2015). Since the condition $T_{e} / T_{i} \ll 1$ is most likely satisfied in low-luminosity disks (Narayan \& Yi 1995; Yuan et al. 2003), this could increase the importance of the presented ion acceleration in these systems.

In terms of the long-term evolution of the acceleration process, in the turbulent environment of accretion disks, we expect many successive $\delta B / B \sim 1$ amplifications and decreases of the field (see also Verscharen et al. 2016). So a more realistic picture of this process should consider the acceleration presented in this work occurring many times as the plasma is gradually accreted. We will study these aspects of the acceleration process in future investigations.

This research was supported by the supercomputing infrastructure of the NLHPC (ECM-02) at the Center for Mathematical Modeling of the University of Chile, the Habanero cluster at Columbia University, and the XSEDE computing system (allocations TG-AST140039, TG-AST140083, and TG-PHY160040). F.L. acknowledges support from NSF grant NST AST-1616037. M.R. acknowledges partial support from FONDECYT through grant FONDECYT REGULAR 1191673. L.S. is thankful for support from DoE DE-SC0016542, NASA ATP NNX-17AG21G, NSF ACI1657507, and NSF AST-1716567. D.V. was supported by the STFC Ernest Rutherford Fellowship ST/P/003826/1.

\section{Appendix A $1 D$ Shear Setup}

The goal of our 1D shear runs is to simulate a shearing plasma, assuming that its properties depend only on the direction parallel to the mean background magnetic field $\langle\boldsymbol{B}\rangle$. This is equivalent to assuming that the wavevectors $\boldsymbol{k}$ captured in the simulations satisfy $\boldsymbol{k} \|\langle\boldsymbol{B}\rangle$. Since, in a shearing plasma, the direction of $\langle\boldsymbol{B}\rangle$ evolves with time, the orientation of the wavevectors that can be consistently resolved needs to evolve accordingly.

Our 1D setup is built upon the "shearing coordinates" setup presented by Riquelme et al. (2012). This setup was designed so that the simulation domain follows the shearing flow of the plasma, which is given by the shear velocity $\boldsymbol{v}(=v \hat{y})$. Figures 1(a) and (b) illustrate the way the shape of the domain evolves as seen by an inertial observer at $t=0$ and $t>0$, respectively. Formally, the shearing coordinates $\left(x^{\prime}, y^{\prime}, z^{\prime}, t^{\prime}\right)$ are defined in terms of the regular, inertial coordinates $(x, y, z, t)$ as

$$
\begin{aligned}
x^{\prime} & =x, \quad y^{\prime}=\Gamma(y-v t), \quad z^{\prime}=z, \quad \text { and } \\
t^{\prime} & =\Gamma\left(t-v y / c^{2}\right),
\end{aligned}
$$

where $\Gamma=\left(1-v^{2} / c^{2}\right)^{-1 / 2}, v=-c \operatorname{arctanh}(s x / c),{ }^{19} c$ is the speed of light, and $s$ is the shear rate of the plasma.

\footnotetext{
19 Notice that, in the limit $|v| \ll c$, the $v=-c \operatorname{arctanh}(s x / c)$ expression is equivalent to $v=-s x$, as we assume in Figures 1(a) and (b). Since $v=-c \operatorname{arctanh}(s x / c)$ ensures that $|v|<c$, this expression was adopted in Riquelme et al. (2012) in order to deduce the equations that describe the dynamics of the plasma in the shearing coordinate system. However, both in Riquelme et al. (2012) and in this paper, we are interested in the plasma dynamics in the regime $|v| \ll c$.
} 
Our 1D domain is defined by $y^{\prime}=0$, and it is shown by the blue lines in Figures 1(a) and (b). These figures show how the length and orientation of the 1D domain change over time. This in turn changes the orientation of the wavevectors $\boldsymbol{k}$ that can be captured within the domain. In addition, there is an initially homogeneous magnetic field, $\boldsymbol{B}$, pointing parallel to the 1D domain at $t=0$. Magnetic flux conservation ensures that $\langle\boldsymbol{B}\rangle$ will always be parallel to the 1D domain at $t>0$, as also depicted in Figures 1(a) and (b). Therefore, if the problem of interest is dominated by waves that propagate parallel to $\langle\boldsymbol{B}\rangle$, our 1D simulations will be able to capture the essence of the phenomenon.

In order for our 1D simulations to consistently satisfy $\boldsymbol{k} \|\langle\boldsymbol{B}\rangle$, we need to replace $x^{\prime}$ with a new coordinate, $x_{1}^{\prime}$, which we define as

$$
x_{1}^{\prime} \equiv x^{\prime}-\frac{y^{\prime} s t^{\prime}}{1+s^{2} t^{\prime 2}}
$$

Indeed, if we assume $|v| \ll c$ and $s y^{\prime} \ll c$, the partial derivatives with respect to the spatial coordinates in the inertial frame are

$$
\begin{aligned}
\frac{\partial}{\partial x} & =\frac{1}{1+s^{2} t^{2}} \frac{\partial}{\partial x_{1}^{\prime}}+s t \frac{\partial}{\partial y^{\prime}}, \\
\frac{\partial}{\partial y} & =\frac{-s t}{1+s^{2} t^{2}} \frac{\partial}{\partial x_{1}^{\prime}}+\frac{\partial}{\partial y^{\prime}}, \text { and } \\
\frac{\partial}{\partial z} & =\frac{\partial}{\partial z^{\prime}} .
\end{aligned}
$$

Thus, if we impose the 1D condition, namely, that the fields depend only on $x_{1}^{\prime}$, with $\partial / \partial y^{\prime}=\partial / \partial z^{\prime}=0$, Equation (11) implies that the gradient of any field component in the inertial frame will have coordinates proportional to the vector $(1,-s t$, $0)$. In the inertial frame, the background magnetic field $\langle\boldsymbol{B}\rangle$ has components $\left(B_{0}, 0,0\right)$ at $t=0$, then, due to magnetic flux freezing, at $t>0$, these components will be $B_{0}(1,-s t, 0)$. Thus, after imposing $\partial / \partial y^{\prime}=\partial / \partial z^{\prime}=0$, our 1D simulations are able to capture all of the modes with wavevectors $\boldsymbol{k}$ parallel to $\langle\boldsymbol{B}\rangle$, despite the fact that $\langle\boldsymbol{B}\rangle$ changes orientation over time.

The assumptions $|v| \ll c$ and $s y^{\prime} \ll c$ are equivalent to assuming that the plasma region of interest has a typical size, $L$, that satisfies $L \ll c / s$. For the study of kinetic instabilities, $L$ is typically of the order of the Larmor radius of the particles. Thus, the restriction $L \ll c / s$ applied to species $j$ becomes $\left[\gamma_{j} s / \omega_{c, j}\right]\left[v_{j, \perp} / c\right] \ll 1$, where $\gamma_{j}, \omega_{c, j}$, and $v_{j, \perp}$ are the Lorentz factor, (nonrelativistic) cyclotron frequency, and velocity perpendicular to $\boldsymbol{B}$ of particles $j$, respectively. In typical astrophysical environments, $\omega_{c, j}$ is many orders of magnitude larger than $s$; therefore, the assumption $L \ll c / s$ is reasonable for the study of kinetic instabilities.

In what follows, we will give expressions for the evolution of fields and particle momenta and positions in our 1D shear setup. The condition $L \ll c / s$ is assumed in all of these expressions.

\section{A.1. Evolution of $\boldsymbol{E}^{\prime}$ and $\boldsymbol{B}^{\prime}$}

Considering the evolution of $\boldsymbol{E}^{\prime}$ and $\boldsymbol{B}^{\prime}$ in terms of $\boldsymbol{J}^{\prime}$ in the shearing coordinates presented in Equations (A14) and (A26) of Riquelme et al. (2012), after replacing $x^{\prime}$ with $x_{1}^{\prime}$ (Equation (10)) and assuming $\partial / \partial y^{\prime}=\partial / \partial z^{\prime}=0$, the field dynamics is given by

$$
\begin{aligned}
\frac{\partial B_{x}^{\prime}}{\partial t^{\prime}} & =c \frac{s t^{\prime}}{1+s^{2} t^{\prime 2}} \frac{\partial E_{z}^{\prime}}{\partial x_{1}^{\prime}}, \frac{\partial B_{y}^{\prime}}{\partial t^{\prime}}=c \frac{1}{1+s^{2} t^{\prime 2}} \frac{\partial E_{z}^{\prime}}{\partial x_{1}^{\prime}}-s B_{x}^{\prime}, \\
\frac{\partial B_{z}^{\prime}}{\partial t^{\prime}} & =\frac{-c}{1+s^{2} t^{\prime 2}}\left(\frac{\partial E_{y}^{\prime}}{\partial x_{1}^{\prime}}+s t^{\prime} \frac{\partial E_{x}^{\prime}}{\partial x_{1}^{\prime}}\right)
\end{aligned}
$$

and

$$
\begin{aligned}
& \frac{\partial E_{x}^{\prime}}{\partial t^{\prime}}=-c \frac{s t^{\prime}}{1+s^{2} t^{\prime 2}} \frac{\partial B_{z}^{\prime}}{\partial x_{1}^{\prime}}-4 \pi J_{x}^{\prime}, \\
& \frac{\partial E_{y}^{\prime}}{\partial t^{\prime}}=-c \frac{1}{1+s^{2} t^{\prime 2}} \frac{\partial B_{z}^{\prime}}{\partial x_{1}^{\prime}}-s E_{x}^{\prime}-4 \pi J_{y}^{\prime}, \\
& \frac{\partial E_{z}^{\prime}}{\partial t^{\prime}}=\frac{c}{1+s^{2} t^{\prime 2}}\left(\frac{\partial B_{y}^{\prime}}{\partial x_{1}^{\prime}}+s t^{\prime} \frac{\partial B_{x}^{\prime}}{\partial x_{1}^{\prime}}\right)-4 \pi J_{z}^{\prime} .
\end{aligned}
$$

In Equations (12) and (13), $\boldsymbol{E}^{\prime}, \boldsymbol{B}^{\prime}$, and $\boldsymbol{J}^{\prime}$, are defined according to the conventional relativistic transformation of the electric field, magnetic field, and current density from the inertial frame to the frame of an observer that moves with velocity $\boldsymbol{v}$ (Equations (A2) and (A28) of Riquelme et al. 2012). This means that $\boldsymbol{J}^{\prime}$ can be calculated directly from the motion of the particles in the shear coordinates, assuming that their momenta $\boldsymbol{p}^{\prime}$ can be obtained from their momenta in the inertial frame $\boldsymbol{p}$, using the conventional relativistic momentum transformation (see Equation (A30) of Riquelme et al. 2012).

\section{A.2. Evolution of Particle Momenta}

Since our 1D setup uses the same definitions of time $\left(t^{\prime}\right)$ and particle momenta $\left(\boldsymbol{p}^{\prime}\right)$ as in the shearing coordinate system of Riquelme et al. (2012), the evolution of $\boldsymbol{p}^{\prime}$ will not change and will be given by

$$
\frac{d \boldsymbol{p}^{\prime}}{d t^{\prime}}=q_{c}\left(\boldsymbol{E}^{\prime}+\frac{\boldsymbol{u}^{\prime}}{c} \times \boldsymbol{B}^{\prime}\right)+s p_{x}^{\prime} \hat{y}
$$

where $q_{c}$ is the particles' electric charge and $\boldsymbol{u}^{\prime}=\boldsymbol{p}^{\prime} / m \gamma^{\prime}$, with $m$ and $\gamma^{\prime}$ being the particle mass and Lorentz factor, respectively. Equation (14) corresponds to Equation (A31) of Riquelme et al. (2012) in the limit $L \ll c / s$.

\section{A.3. Evolution of Particle Positions}

By taking the derivative of Equation (10) with respect to $t^{\prime}$ and applying the limit $L \ll c / s$ to the expressions for $d x^{\prime} / d t^{\prime}$ and $d y^{\prime} / d t^{\prime}$ given by Equation (A35) of Riquelme et al. (2012), we find

$$
\frac{d x_{1}^{\prime}}{d t^{\prime}}=u_{x}^{\prime} \frac{1}{1+s^{2} t^{\prime 2}}-u_{y}^{\prime} \frac{s t^{\prime}}{1+s^{2} t^{\prime 2}},
$$

where $u_{x}^{\prime}$ and $u_{y}^{\prime}$ are the $x$ and $y$ components of $\boldsymbol{u}^{\prime}$. Equation (15) implies that $d x_{1}^{\prime} / d t^{\prime}$ is the scalar product between $\boldsymbol{u}^{\prime}$ and $\langle\boldsymbol{B}\rangle /|\langle\boldsymbol{B}\rangle|\left(=\left(\hat{x}-s t^{\prime} \hat{y}\right) /\left(1+s^{2} t^{\prime 2}\right)^{1 / 2}\right)$, corrected by the "expansion" of the 1D domain (represented by the $y^{\prime}=0$ region depicted in blue in Figures 1(a) and (b)), which provides the extra common factor $1 /\left(1+s^{2} t^{\prime 2}\right)^{1 / 2}$ on the right-hand side of Equation (15). This implies that $x_{1}^{\prime}$ correctly represents particle displacements along $\langle\boldsymbol{B}\rangle$. 


\section{A.4. Charge Conservation}

The evolution of particle positions in our simulations is consistent with the conservation of electric charge density. This can be checked by considering Gauss's law in the inertial frame: $\nabla \cdot \boldsymbol{E}=4 \pi \rho_{c}$, where $\boldsymbol{E}$ and $\rho_{c}$ are the electric field and charge density in that frame. Indeed, applying the transformations given by Equation (9) to Gauss's law in the limit $L \ll c / s$, and assuming first the more general problem in $3 \mathrm{D}$ described in Riquelme et al. (2012),

$$
\frac{\partial \rho_{c}^{\prime}}{\partial t^{\prime}}+\frac{\partial J_{x}^{\prime}}{\partial x^{\prime}}+\frac{\partial\left(J_{y}^{\prime}+s t^{\prime} J_{x}^{\prime}\right)}{\partial y^{\prime}}+\frac{\partial J_{z}^{\prime}}{\partial z^{\prime}}=0,
$$

where $\rho_{c}^{\prime}$ is the charge density of the plasma calculated using the shearing coordinates, which, as shown in Equation (A29) of Riquelme et al. (2012), is equal to $\rho_{c}$ in the limit $L \ll c / s$. Thus, according to Equation (16), in order to satisfy charge conservation in our multidimensional shearing coordinate, particle motions in $y^{\prime}$ lead to an effective current along the $y^{\prime}$ direction of $J_{y}^{\prime}+s t^{\prime} J_{x}^{\prime}$. Thus, charge conservation is ensured in our multidimensional shearing coordinate simulations by evolving $x^{\prime}, y^{\prime}$, and $z^{\prime}$ according to

$$
\frac{d x^{\prime}}{d t^{\prime}}=u_{x}^{\prime}, \quad \frac{d y^{\prime}}{d t^{\prime}}=u_{y}^{\prime}+s t^{\prime} u_{x}^{\prime}, \quad \text { and } \frac{d z^{\prime}}{d t^{\prime}}=u_{z}^{\prime},
$$

which we do in the 2D simulations presented in this paper and in our previous works (e.g., Riquelme et al. 2017). Equation (17) is equivalent to Equation (A35) of Riquelme et al. (2012) in the limit $L \ll c / s$. $^{20}$

Finally, by making an analogous analysis in the case of the 1D setup, we show that charge conservation implies

$$
\frac{\partial \rho_{c}^{\prime}}{\partial t^{\prime}}+\frac{\partial}{\partial x_{1}^{\prime}}\left(\frac{J_{x}^{\prime}}{1+s^{2} t^{\prime 2}}-\frac{s t^{\prime} J_{y}^{\prime}}{1+s^{2} t^{\prime 2}}\right)=0
$$

Thus, according to Equation (18), in order for our 1D runs to conserve charge, $x_{1}^{\prime}$ must evolve so that the effective current along $\langle\boldsymbol{B}\rangle$ is $\left(J_{x}^{\prime}-s t^{\prime} J_{y}^{\prime}\right) /\left(1+s^{2} t^{\prime 2}\right)$, which is indeed satisfied by the evolution of $x_{1}^{\prime}$ given by Equation (15).

\section{Appendix B \\ IC versus Mirror under Realistic Conditions: Linear Theory Analysis}

In order to inquire whether the dominance of the IC instability for $\beta_{i}^{\text {init }} \lesssim 1$ continues to be valid in realistic, astrophysically relevant regimes $\left(m_{i} / m_{e}=1836\right.$ and $\omega_{c, i} / s \gg 800$, which we cannot study with our 2D simulations), we make use of linear theory. Thus, we calculate the threshold ion pressure anisotropy, $\Delta p_{i} / p_{\|, i} \equiv\left(p_{\perp, i}-p_{\|, i}\right) / p_{\|, i}$, needed for the growth of IC and mirror modes at a rate $s$, using the linear Vlasov solver NHDS (Verscharen \& Chandran 2018, which assumes bi-Maxwellian, nonrelativistic ion velocity distributions). ${ }^{21}$ This condition is motivated by the assumption that, in order to maintain $\Delta p_{i} / p_{\|, i}$ at a nearly stationary level (as we see in Figures 15(a) and (b)),

\footnotetext{
${ }^{20}$ Notice that the notation used in Equation (A35) of Riquelme et al. (2012) is different from the one used in this work, and that we interpret their $u_{x}^{\prime}, u_{y}^{\prime}$, and $u_{z}^{\prime}$ as our $d x^{\prime} / d t^{\prime}, d y^{\prime} / d t^{\prime}$, and $d z^{\prime} / d t^{\prime}$.

21 Modern linear solvers to the linear Vlasov-Maxwell system of equations can account for relativistic effects and non-Maxwellian background distributions (Verscharen et al. 2018). Studies using this type of solver can evaluate these effects; however, their application is beyond the scope of this paper.
}

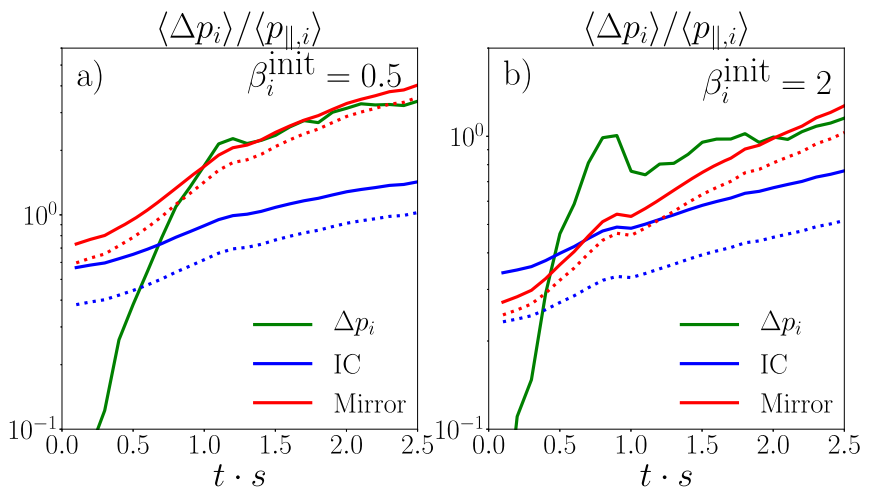

Figure 15. Mirror (solid red) and IC (solid blue) linear anisotropy thresholds (assuming growth rate $s$ ) in $2 \mathrm{D}$ runs $\mathrm{S} 2 \mathrm{~m} 2 \mathrm{~b} 0.5\left(m_{i} / m_{e}=2\right.$ and $\beta_{i}^{\text {init }}=0.5$; panel (a)) and S2m2b2 ( $m_{i} / m_{e}=2$ and $\beta_{i}^{\text {init }}=2$; panel (b)). The anisotropies obtained directly from the simulations are in solid green. The dotted lines show analogous linear anisotropy thresholds for the mirror (dotted red) and IC (dotted blue) instabilities, assuming $m_{i} / m_{e}=1836$ and a growth rate equal to $10^{-6} \omega_{c, i}$.

the modes that provide the pitch-angle scattering must grow at roughly the rate at which the anisotropy is driven, which is $\sim s$. Thus, if nonlinear effects did not play any significant role, the dominant instability should be the one with the lowest theoretical anisotropy threshold for a given value of $s$.

However, nonlinear effects are expected to be important and to affect the IC and mirror instabilities differently. Indeed, in situ observations in the solar wind show significant discrepancies between linear theory and the measured ion anisotropy in regions of parameter space in which the IC instability should dominate (Hellinger et al. 2006; Bale et al. 2009). One possible explanation is the departure from biMaxwellian ion velocity distributions observed in the solar wind, which may affect the efficiency of the resonant scattering between ions and the IC modes (Isenberg et al. 2013). Another possibility is the inhomogeneity in the magnetic field produced by the (subdominant but still present) mirror modes, which may also affect this resonance (Southwood \& Kivelson 1993). Our approach is thus to estimate these nonlinear effects using the simulated cases. These simulations thus provide us with a calibration of the linear theory criterion for determining the dominant instability, which can then be applied to astrophysically realistic regimes.

Figures 15(a) and (b) show the linear anisotropy threshold given by the mirror (solid red) and IC (solid blue) instabilities with growth rate $s$ in runs $\mathrm{S} 2 \mathrm{~m} 2 \mathrm{~b} 0.5$ and $\mathrm{S} 2 \mathrm{~m} 2 \mathrm{~b} 2$, which have $\beta_{i}^{\text {init }}=0.5$ and 2 , respectively (both with $m_{i} / m_{e}=2$ and $\left.\omega_{c, i}^{\text {init }} / s=800\right)$, and compare them with the anisotropies obtained from the simulations (solid green). First, in the ICdominated regime (run $\mathrm{S} 2 \mathrm{~m} 2 \mathrm{~b} 0.5$ ), the IC threshold is $\sim$ three times smaller than the ion anisotropy obtained from the simulation, showing that, in the case of the IC instability, nonlinear effects give rise to ion anisotropies that are significantly larger than what is implied by the linear theory threshold. Also, the IC threshold is at least $\sim$ two times smaller than the mirror threshold (Figure 15(a)), while in the mirrordominated case (Figure 15(b)), the IC threshold is at most $\sim 1.5$ times smaller. These results suggest that, in order for the IC instability to dominate, the IC threshold should be at least $\sim$ two times smaller than the mirror threshold.

We thus apply this criterion to astrophysically realistic cases, in which $m_{i} / m_{e}=1836$ and the instabilities grow at a rate of 
$10^{-6} \omega_{c, i}$. Figures 15(a) and (b) show in dotted lines the corresponding linear anisotropy thresholds for the mirror (red) and IC (blue) instabilities for $\beta_{i}^{\text {init }}=0.5$ and 2, respectively. In the $\beta_{i}^{\text {init }}=0.5$ case, the IC threshold is always at least $\sim$ three times smaller than the mirror threshold, implying that, under realistic conditions, the IC modes continue to dominate in the $\beta_{i}^{\text {init }}=0.5$ case. In $\beta_{i}^{\text {init }}=2$, on the other hand, the linear IC threshold is smaller than the mirror threshold by a factor of $\lesssim 2$ during most of the simulations. Since we estimate that, in order for the IC instability to dominate, the mirror anisotropy threshold should be at least $\sim$ two times larger than that of the IC modes, this suggests that the mirror instability continues to dominate in this case.

\section{ORCID iDs}

Mario Riquelme (1) https://orcid.org/0000-0003-2928-6412

Daniel Verscharen (1D https://orcid.org/0000-0002-0497-1096

\section{References}

Anderson, B. J., Fuselier, S. A., \& Murr, D. 1991, GeoRL, 18, 1955 Bale, S. D., Kasper, J. C., Howes, G. G., et al. 2009, PRL, 103, 211101 Buneman, O. 1993, in Computer Space Plasma Physics, ed. H. Matsumoto \& Y. Omura (Tokyo: Terra Scientific), 67

Chael, A., Rowan, M., Narayan, R., Johnson, M., \& Sironi, L. 2018, MNRAS, 478, 5209

Chandran, B. 2003, ApJ, 599, 1426

Chew, G. F., Goldberger, M. L., \& Low, F. E. 1956, RSPSA, 236, 112

Cho, J., \& Lazarian, A. 2006, ApJ, 638, 811

Dermer, C. D., Miller, J. A., \& Li, H. 1996, ApJ, 456, 106

Gary, S. P. 1992, JGR, 97, 8519

Gary, S. P., \& Wang, J. 1996, JGR, 101, 10749

Hasegawa, A. 1969, PhFl, 12, 2642
Hellinger, P., Travnicek, P., Kasper, J. C., \& Lazarus, A. J. 2006, GeoRL, 33, L09101

Isenberg, P. A., Maruca, B. A., \& Kasper, J. C. 2013, ApJ, 773, 164

Kulsrud, R. M. 1983, in Handbook of Plasma Physics, ed. M. N. Rosenbluth \& R. Z. Sagdeev (Amsterdam: North-Holland), 115

Kulsrud, R. M., \& Pearce, W. P. 1969, ApJ, 156, 445

Lynn, J. W., Quataert, E., Chandran, B. D. G., \& Parrish, I. J. 2014, ApJ, 791, 71

Lyutikov, M. 2007, ApJL, 668, L1

Maruca, B. A., Kasper, J. C., \& Bale, S. D. 2011, PhRvL, 107, 201101

Narayan, R., \& Yi, I. 1995, ApJ, 452, 710

Petrosian, V., \& Liu, S. 2004, ApJ, 610, 550

Pokhotelov, O. A., Sagdeev, R. Z., Balikhin, M. A., \& Treuman, R. A. 2004, JGR, 109, A09213

Ponti, G., George, E., Scaringi, S., et al. 2017, MNRAS, 468, 2447

Remya, B., Reddy, R. V., Tsurutani, B. T., Lakhina, G. S., \& Echer, E. 2013, JGR, 118, 785

Riquelme, M., Osorio, A., \& Quataert, E. 2017, ApJ, 850, 113

Riquelme, M., Quataert, E., Sharma, P., \& Spitkovsky, A. 2012, ApJ, 755, 50

Riquelme, M., Quataert, E., \& Verscharen, D. 2016, ApJ, 824, 123

Schekochihin, A. A., Cowley, S. C., Kulsrud, R. M., Hammett, G. W., \& Sharma, P. 2005, ApJ, 629, 139

Sharma, P., Hammett, G. W., Quataert, E., \& Stone, J. 2006, ApJ, 637, 952

Sharma, P., Quataert, E., Hammett, G. W., \& Stone, J. 2007, ApJ, 667, 714

Sironi, L. 2015, ApJ, 800, 89

Sironi, L., \& Narayan, R. 2015, ApJ, 800, 88

Snyder, P. B., Hammett, G. W., \& Dorland, W. 1997, PhPl, 4, 3974

Southwood, D. J., \& Kivelson, M. G. 1993, JGR, 98, 9181

Spitkovsky, A. 2005, in AIP Conf. Proc. 801, Astrophysical Soures of High Energy Particles and Radiation, ed. T. Bulik, B. Rudak, \& G. Madejski (San Francisco, CA: ASP), 345

Verscharen, D., \& Chandran, B. 2018, RNAAS, 2, 13

Verscharen, D., Chandran, B., Klein, K. G., \& Quataert, E. 2016, ApJ, 831,128

Verscharen, D., Klein, K. G., Chandran, B. D. G., et al. 2018, JPIPh, 84 , 905840403

Verscharen, D., Klein, K. G., \& Maruca, B. A. 2019, arXiv:1902.03448

Yuan, F., Quataert, E., \& Narayan, R. 2003, ApJ, 598, 301 